Sharif University of Technology
Scientia Iranica
SCIENTIA $\quad \begin{gathered}\text { Transactions D: Computer Science \& Engineering and Electrical Engineering } \\ \text { w }\end{gathered}$

Invited/Review Article

\title{
A survey of bandgap and non-bandgap based voltage reference techniques
}

\author{
V. Hande* and M. Shojaei Baghini \\ Department of Electrical Engineering, Indian Institute of Technology (IIT)-Bombay, Mumbai, MH, India. \\ Received 10 April 2016; received in revised form 1 July 2016; accepted 29 October 2016
}

\author{
KEYWORDS \\ Bandgap; \\ Sub-1 V; \\ Survey; \\ Temperature \\ coefficient; \\ Voltage reference.
}

\begin{abstract}
The design challenges of voltage reference generators in CMOS technology have increased over the years in low-voltage low-power CMOS integrated circuits, constituting analog, digital, and mixed-signal modules. The emergence of hand-held power autonomous devices pushes the power consumption limit to $n W$ regime. Along with these confrontations, limited full-scale range of data converters at low supply levels demands accurate reference voltage generators. This paper reviews the allied design challenges and discusses the evolved methodologies to tackle them. This paper also prominently surveys the sub- $1 \mathrm{~V}$ voltage reference topologies presented in the literature along with classic bandgap based voltage reference topologies. Non-bandgap (only CMOS) based reference architectures are proven to be area- and power-efficient, but always have to be accompanied with auxiliary on/off chip trimming mechanism for high accuracy. We also provide insightful analysis of the voltage reference topologies required by the designers.
\end{abstract}

(C) 2016 Sharif University of Technology. All rights reserved.

\section{Introduction}

An accurate voltage reference is an important block in most of the Integrated Circuits (ICs). It establishes a reference voltage point for the rest of the circuit blocks in a complete system to achieve reliable and predictable performances. It can be used with a regulator to build a power-supply, in an operational amplifier to set up bias voltages, and/or in data converters to establish a standard voltage for comparison. The accuracy of the reference voltage often decides on the overall performance of a system.

Operating principle of the circuit adds a voltage that decreases linearly with temperature to one that increases linearly with temperature to produce a reference voltage that is constant with respect to temperature in the first order. The base-emitter

*. Corresponding author.

E-mail addresses: vinayak.hande@iitb.ac.in (V. Hande); mshojaei@ee.iitb.ac.in (M. ShojaeiBaghini) voltage $\left(V_{\mathrm{BE}}\right)$ of a bipolar transistor decreases linearly with temperature, i.e. it has a Complementary-ToAbsolute-Temperature (CTAT) dependence. The voltage that increases linearly with temperature, i.e., the Proportional-To-Absolute-Temperature (PTAT) voltage, is produced through the difference in the values of $V_{\mathrm{BE}}$ in two bipolar transistors operating under different current densities. A bandgap reference circuit adds these CTAT and PTAT voltages to produce a temperature-invariant voltage $V_{\mathrm{REF}}$. The value of the reference voltage is close to the bandgap voltage of silicon $(1.2 \mathrm{~V})$.

This paper is a survey of voltage reference topologies since they were presented in the year 1971. We would like to explain the terminology, in which bandgap/sub-bandgap voltage references are the topologies that give the output voltage $\approx 1.23 \mathrm{~V}$ or up/downscaled version of that. Also, non-bandgap voltage references or voltage references generally do not constitute any bi-polar device/s. Therefore, these voltage reference circuits are not bound to give $\approx 1.23 \mathrm{~V}$ 
or scaled versions of $1.23 \mathrm{~V}$. That means, non-bandgap voltage references mainly have only MOSFETs and/or resistors. The paper is basically divided into three parts. In the first part, we have discussed the classic reference generator topologies. These topologies are generally bandgap based. The second part of the paper deals with the reference topologies broadly proposed after 1997. Researchers started working on sub-1 V bandgap voltage as technology started shrinking and resulted in drastic fall of operating supply voltage. Finally, the third part of the paper discusses the curvature correction techniques that improve the accuracy.

\section{Definition of performance indices}

There are several different performance indices used to specify and compare voltage references. They are listed below:

1. Temperature Coefficient (TC);

2. Minimum operable supply voltage;

3. Power consumption;

4. Noise;

5. Line Sensitivity (LS);

6. Power Supply Noise Attenuation (PSNA);

7. Area consumption.

Some of these parameters like area, power consumption, and minimum supply voltage are quiet straight- forward, while others require explanations. Many of the indices are vulnerable to the characteristics and variations of the fabrication process.

Actually, the TC of a voltage reference has been presented in many ways. However, researchers commonly use the effective Temperature Coefficient (TC). It is given in Eq. (1). It measures the maximum variation of the voltage reference across a specific temperature range $\Delta T$ and normalizes this value against the nominal $V_{\mathrm{REF}}$ at room temperature. Its unit is $\mathrm{ppm} /{ }^{\circ} \mathrm{C}$.

$$
T C=\frac{V_{\mathrm{REF}_{\max }}-V_{\mathrm{REF}_{\min }}}{V_{\mathrm{REF}_{\mathrm{nom}}} \Delta T}
$$

Another important performance index is the ability of the voltage reference to reject supply variations. This measurement at DC is called Line Sensitivity (LS) or Line Regulation (LR), given by Eq. (2), and measured in $\mathrm{mV} / \mathrm{V}$. Its frequency behavior is given by Eq. (3), called Power Supply Noise Attenuation or PSNA [1], and measured in $\mathrm{dB}$. The PSNA response is primarily evaluated at two frequencies, i.e. $100 \mathrm{~Hz}$ and $1 \mathrm{MHz}$ (as low-frequency and high-frequency responses).

$$
\begin{aligned}
& \mathrm{LS}=\frac{V_{\mathrm{REF}_{\text {max }}}-V_{\mathrm{REF}_{\text {min }}}}{\Delta V_{D D}} \\
& \operatorname{PSNA}(\omega)=\frac{V_{R E F}(w)}{V_{D D}(w)}
\end{aligned}
$$

As with every electronic circuit, the intrinsic device noise is one of the limiting accuracy factors. For recent voltage references, its power spectral density is usually specified at $100 \mathrm{~Hz}$ (low frequency) for comparison purposes, while the total RMS noise depends on the chosen bandwidth. Noise parameters are not discussed here, as all the mentioned papers did not mentioned it specifically.

Above discussed are the main performance indices used to specify and compare voltage references. We will use them extensively in the review of recent circuits. Besides the obvious design considerations, there are other design considerations in a voltage reference circuit that are important and that, at the same time, also affect the above performance parameters. Among these are circuit size and power dissipation. These two are becoming more important parameters as hand-held devices are becoming popular. We have extensively considered these parameters for our study.

\section{Classic voltage references}

One of the first bandgap voltage reference circuits was presented by Robert Widlar of the National Semiconductor in 1971. It is well-known as the Widlar's bandgap voltage reference circuit [2]. The Widlar bandgap voltage reference circuit was implemented with the conventional bipolar technology, of which the complete circuit is shown in Figure 1.

The Widlar bandgap voltage reference circuit gen-

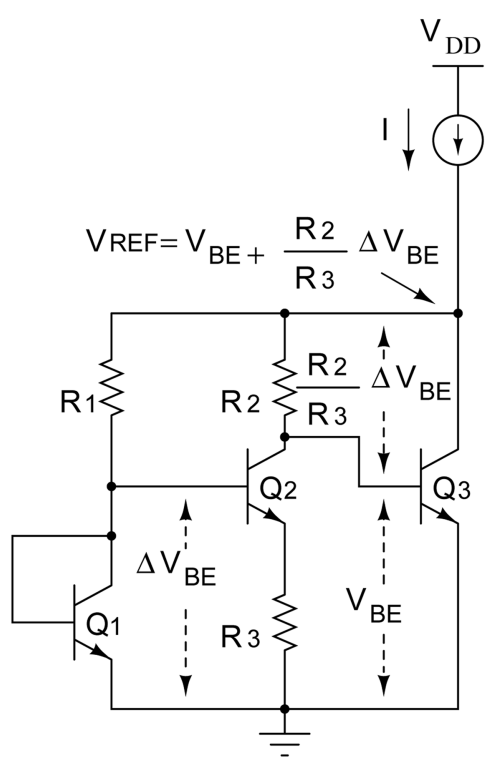

Figure 1. Widlar voltage reference: 1971. 
erates a stable low-TC reference voltage at $1.23 \mathrm{~V}$. This implementation of a bandgap voltage reference circuit was successfully applied in the National Semiconductors voltage regulator integrated circuit LM 113, which proved capable of achieving an output voltage with low TC. Since then, it has been applied in many voltage regulator ICs to generate the internal reference voltage/s.

\subsection{Robert Widlar's voltage reference: 1971}

One of the oldest $V_{\mathrm{BE}}$ and $\Delta V_{\mathrm{BE}}$ compensated bandgap voltage reference circuits is the Widlar bandgap voltage reference circuit introduced by Robert Widlar in 1971. These bandgap circuits are based on the principle shown in Figure 1 . The transistors $Q_{1}$ and $Q_{2}$ are operated at different current densities to produce temperature-proportional voltages across $R_{3}$ and $R_{2}$. A third transistor $Q_{3}$ is used to sense the output voltage through $R_{2}$. As a result, $Q_{3}$ drives the output to a voltage which is the sum of $V_{\mathrm{BE}}$ of $Q_{3}$ and the temperature-dependent voltage across $R_{2}$. When the output voltage is set to approximate the bandgap voltage of silicon, the voltage across $R_{2}$ will compensate the temperature coefficient of $V_{\mathrm{BE}}$, and the output voltage will be temperature-invariant.

Furthermore, the thermal voltage $\left(V_{T}\right)$ extracted from the $\Delta V_{\mathrm{BE}}$ of two BJTs with different emitter areas and, hence, different current densities will form the PTAT voltage, while the base-emitter voltage $\left(V_{\mathrm{BE}}\right)$ of the BJT will form the CTAT voltage. The circuit is simple, but not easy to implement in modern CMOS process or to control the output, because the BJTs implemented in the CMOS process are sensitive to process variation.

In this circuit, $Q_{1}$ is operated at a relatively high current density. The current density of $Q_{2}$ is about 10 times lower and the emitter-base voltage difference $\Delta V_{\mathrm{BE}}$ between the two devices appears across $R_{3}$, defining the emitter current and the collector current if the transistor gain is high. $Q_{3}$ then works as a current gain stage, also providing the $V_{\mathrm{BE}}$ voltage necessary to form the output. A simplified analysis of the circuit follows.

The collector current IC of a BJT is written as:

$$
I_{C}=I_{0}\left[\exp \left(\frac{V_{\mathrm{BE}}}{\eta V_{T}}-1\right)\right] \text {. }
$$

where $I_{0}$ is a process-dependent constant, $V_{\mathrm{BE}}$ is the base-emitter voltage, $\eta$ is the emission coefficient, and $V_{T}$ is the thermal voltage. If two transistors are operated with different collector current densities, $J_{i}$, then the difference of their base-emitter voltages is given by Eq. (5).

$$
\Delta V_{\mathrm{BE}}=V_{T} \ln \left(\frac{J_{1}}{J_{2}}\right) .
$$

In Figure 1, one can see that the output of the bandgap reference (BGR) proposed by Widlar is given by the sum of the base-emitter voltage of $Q_{3}$ and the multiplied PTAT term on $R_{2}$, as shown by Eq. (6):

$$
V_{\mathrm{REF}}=V_{\mathrm{BE}}+\frac{R_{2}}{R_{3}} \Delta V_{\mathrm{BE}}
$$

For the time being, we consider that $V_{\mathrm{BE}}$ can be expressed by the linear expression $V_{\mathrm{BE}}=V_{\mathrm{BG}} C T$, where $V_{\mathrm{BG}}$ is the silicon bandgap voltage at $0 \mathrm{~K}$, $C$ is a constant, and $T$ is the absolute temperature. Other higher-order temperature effects will be studied extensively in Section 7 . Therefore, $R_{1}$ and $R_{2}$ can be scaled together with the current density difference of $Q_{1}$ and $Q_{2}$ to achieve $d V_{\mathrm{REF}} / d T=0$.

\subsection{Kuijk's voltage reference: 1973}

Among the various existing bandgap voltage reference architectures to date, one of the most ubiquitous ones is the Kuijk bandgap reference circuit, shown in Figure 2 [3]. Reference voltage $V_{\mathrm{REF}}$ is evaluated as the sum of the voltage drops across $R_{1}, R_{3}$, and the base-emitter voltage of $D_{1}\left(V_{\mathrm{BE}, 1}\right)$. If opamp gain is very high, reference voltage is:

$$
V_{\mathrm{REF}}=V_{\mathrm{BE}}+V_{T}\left(1+\frac{R_{1}}{R_{3}}\right) \ln \left(\frac{I_{S 1} R_{1}}{I_{S 2} R_{2}}\right),
$$

where $I_{S 1}$ and $I_{S 2}$ represent the saturation currents of $D_{1}$ and $D_{2}$. The first term in Eq. (7) is Complementary To Absolute Temperature (CTAT) and the second term is $V_{T}$-based, i.e. Proportional To Absolute Temperature (PTAT). The sum of both is trimmed with scaling factor (selecting proper resistor values) to achieve temperature-insensitive reference voltage.

\subsection{Brokaw's voltage reference: 1974}

The important fact is that the Widlar's circuit neglects the effect of base current flowing in $R_{1}$ and $R_{2}$. The variability in this current due to processing and temperature effects on beta (feed-forward current gain)

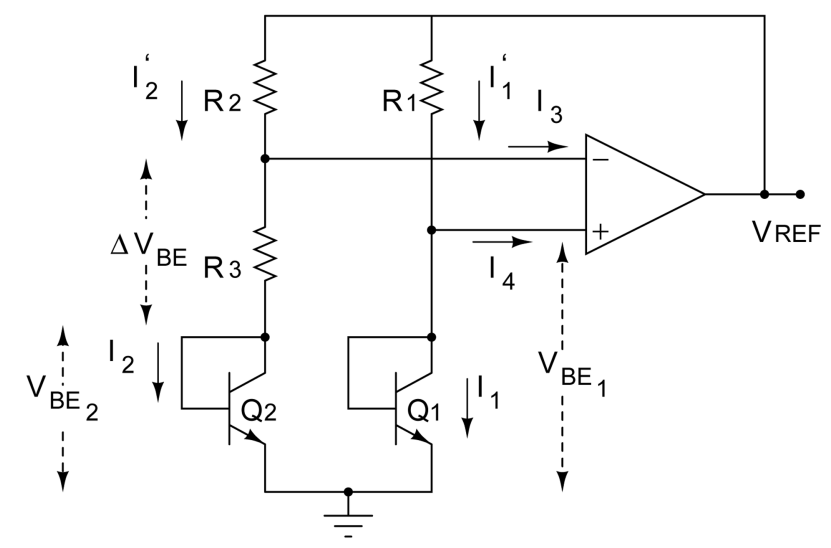

Figure 2. Kuijk voltage reference: 1973. 


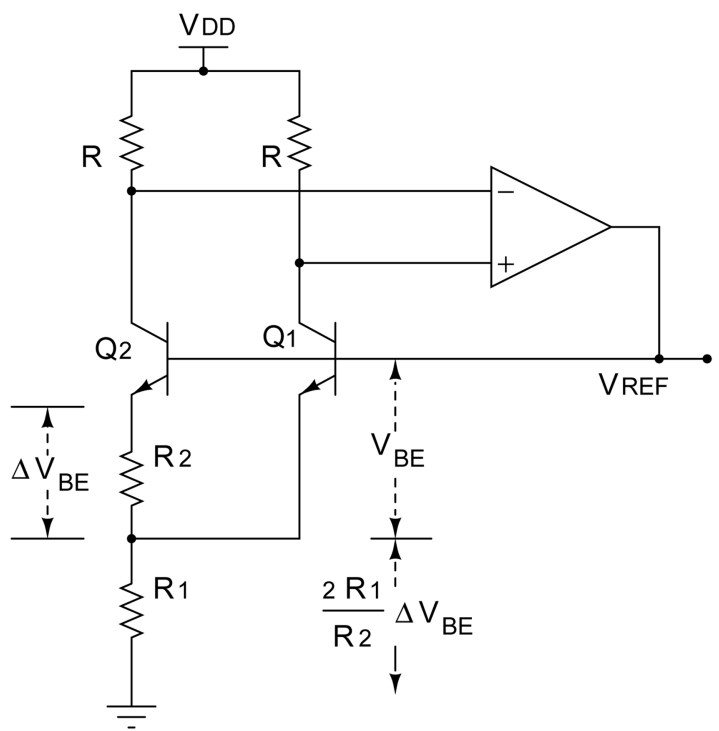

Figure 3. Brokaw voltage reference: 1974 .

gives rise to an output voltage error and drift. This effect is particularly severe when the current in $D_{1}$ is made much smaller than currents in $D_{1}$ and $D_{2}$ to produce the required current density difference. To resolve this issue, Paul Brokaw introduced the wellknown BGR circuit, shown in Figure 3 [4].

The Brokaw bandgap voltage reference circuit is simple and the compensation performance can be easily adjusted by post-processing, such as trimming resistors $R_{1}$ and $R_{2}$, thus overcoming the process variation problem. On the contrary, trimming resistors $R_{1}$ and $R_{2}$ in the Widlar bandgap voltage reference circuit alter the currents flowing through the BJTs and, hence, the performance of the voltage reference circuit. As a result, adjusting performance of the Widlar bandgap voltage reference circuit by trimming is not easy to implement. Nevertheless, a Brokaw bandgap voltage reference circuit that applies an opamp has the advantage of a large output driving power, which makes it suitable to be applied in circuits with heavy loads, while the Widlar bandgap voltage reference circuit requires an extra buffer stage to provide the driving power for large loads.

In this circuit, the operational amplifier makes $I C_{1}=I C_{2}$ through resistances $R$, and the $\Delta V_{\mathrm{BE}}$ that appears across $R_{2}$ is then multiplied by $2 R_{1}$, since both emitter currents of $Q_{1}$ and $Q_{2}$ flow into this resistor. The reference output is the sum of $V_{\mathrm{BE}}, Q_{1}$, and $V R_{1}$. The author also introduces some compensation schemes for the base currents of $Q_{1}-Q_{2}$ through the use of auxiliary base resistances, not shown in Figure 3.

\section{Sub-1 V voltage references}

The IC fabrication trend shows reduction in channel length, which leads to overall decrement in the geom- etry size of transistor. Reduction in the geometry size leads to increase in circuit capacity and speed. As transistor size decreases, the circuit functionality of a given area of substrate increases. Smaller device size also yields lower parasitic capacitance, which increases speed and decreases power consumption. Simultaneously, operating supply voltage must also be scaled down due to the increased electric field and reduced breakdown voltage caused by the higher doping profile required by small device. Decreased operating voltage facilitates low power consumption, which is increasingly important as circuit complexity increases. However, the voltage reference circuit becomes more difficult to design with the lower supply voltage.

The low operating voltage imposes two design challenges on voltage reference circuit designing. The first constraint is the output of the voltage reference circuit. The output voltage of the voltage reference circuit must be lower than the supply voltage. The output voltage of most of the conventional bandgap voltage reference circuits discussed in Section 3 is $1.23 \mathrm{~V}$. When the supply voltage is lowered to below $1 \mathrm{~V}$, how can one achieve a reference voltage with magnitude below $1 \mathrm{~V}$. The voltage reference circuit capable of generating a lower output voltage is referred to as sub-1 V voltage reference circuit, which literally means that the output of the voltage reference circuit is lower than $1 \mathrm{~V}$. The second design challenge is the operating supply voltage. The voltage reference circuit is required to be able to work with a low supply voltage. When the voltage reference circuit can operate at a supply voltage lower than $1 \mathrm{~V}$, it is known as sub$1 \mathrm{~V}$ supply voltage reference circuit. Obviously, a sub$1 \mathrm{~V}$ supply voltage reference circuit can only generate reference voltage that is lower than the supply voltage. As a result, it is also a sub- $1 \mathrm{~V}$ voltage reference circuit.

The conventional bandgap voltage reference circuits presented in Section 3 are suitable neither for low voltage applications nor for generating low reference voltage. The constraint on achieving a sub-1 V reference voltage is the bandgap voltage itself. In the subsequent section, we study some of the popular sub$1 \mathrm{~V}$ voltage reference generation circuits.

\subsection{Neuteboom's voltage reference: 1997}

While designing a DSP-based hearing instrument integrated circuit, Neuteboom et al. [5] were limited with supply voltage of $0.9 \mathrm{~V}$. It was clear that with this limited supply voltage of $0.9 \mathrm{~V}$, a conventional reference voltage of $1.23 \mathrm{~V}$ could not be realized. Neuteboom et al. proposed the reference circuit illustrated in Figure 4, which is based on the resistive division technique and provides an output voltage lower than $1.23 \mathrm{~V}$. Their reference circuit makes use of three vertical PNP transistors with emitter ratio of $1: N: N$. The opamp controls the emitter current and maintains a $\Delta V_{\mathrm{BE}}$ 


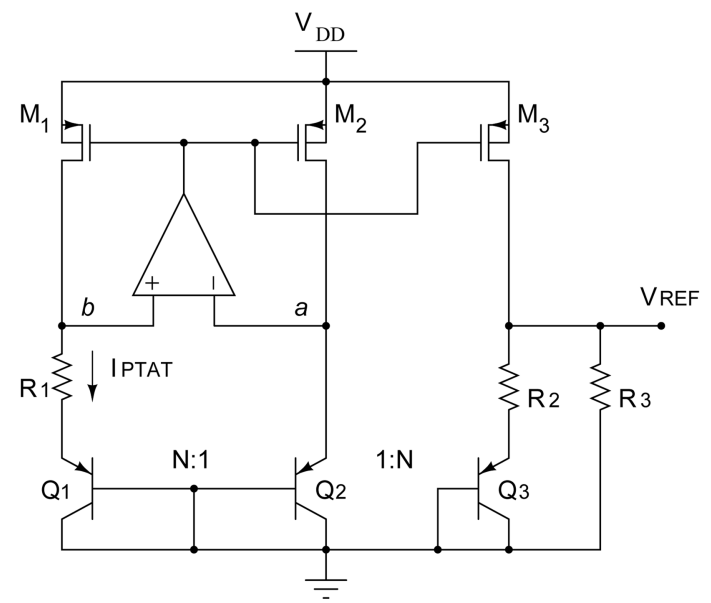

Figure 4. Neuteboom voltage reference: 1997.

across the resistor $R_{1}$ in the PTAT current generation loop. Operation under low supply voltage operation condition is achieved by connecting the resistor $R_{3}$ across the bandgap reference, and the resulting output voltage becomes a fraction of the bandgap voltage, which is given by:

$$
V_{\mathrm{REF}}=\frac{R_{3}}{R_{2}+R_{3}}\left(V_{\mathrm{BE}}+I_{\mathrm{PTAT}} R_{2}\right),
$$

that can be scaled to any value. A down-scaling of the bandgap voltage is obtained by proper choice of resistors $R_{2}$ and $R_{3}$. The proposed circuit achieves an output voltage of $0.67 \mathrm{~V}$ under a minimum supply voltage of $0.9 \mathrm{~V}$. Basically, the result is a simple resistive division of the bandgap reference voltage, i.e. $1.23 \mathrm{~V}$.

\subsection{Banba's voltage reference: 1999}

Similar to Neuteboom et al. [5], Banba et al. [6] have also proposed a technique to overcome these limitations to implement sub-1 $\mathrm{V}$ bandgap voltage reference circuits in CMOS technology. They have used resistive sub-division technique to reduce the minimum required supply voltage. The operation of the sub-1 V opamp based bandgap voltage reference circuit with resistive division is similar to that of the conventional opamp based bandgap voltage reference circuit, where an opamp will form an inverted feedback loop to force the two input nodes of the opamp to have the same voltages. Consider the case of $R_{2}=R_{3}$, such that $I_{1}=I_{2} ; N$ is the emitter area ratio between $Q_{1}$ and $Q_{2}$. The current $I_{2}$ is mirrored to form $I_{3}$ and, therefore, the output voltage of the bandgap voltage reference circuit is given by:

$$
V_{\mathrm{REF}}=\frac{R_{4}}{R_{3}}\left(V_{\mathrm{BE}_{1}}+\frac{R_{3}}{R_{1}} V_{T} \ln (N)\right) .
$$

It can be observed that the performance of this voltage reference circuit should be comparable to that

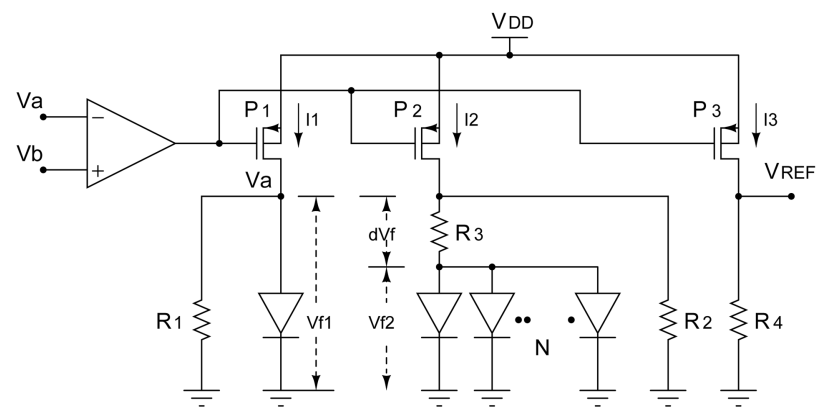

Figure 5. Banba voltage reference: 1999.

of the conventional bandgap voltage reference circuit, at least in theory. At the same time, we should also notice that $V_{\text {REF }}$ can be scaled by the resistor ratio $R_{4} / R_{3}$, thus, achieving an arbitrary $V_{\mathrm{REF}}$.

As observed from Figure 5, the minimum operating supply voltage of such a circuit is found to be $1.4 \mathrm{~V}$, which is almost the same as that of the conventional bandgap voltage reference circuit. This is due to the limitation of the input common mode of the opamp. This limitation makes the sub-1 $\mathrm{V}$ bandgap voltage reference circuit with resistive division not applicable for low supply voltage applications. Moreover, susceptibility of the bandgap voltage reference circuit to noise increases because of the low output impedance of the bandgap voltage reference circuit, which is basically the output impedance of $M_{3}$. Finally, the transistor, $M_{3}$, has to be biased in saturation mode to form a proper current mirror; thus, the resistor value of $R_{4}$ will be confined to a certain region of values. The last, but not least, important fact is that the load regulation of the circuit is very low because of the low output impedance of the voltage reference circuit, which equals the output impedance of the current mirror.

Nevertheless, the resistive division technique does demonstrate that there are circuit topologies that can alleviate the common-mode input voltage constraint of the opamp in the reference circuit and, hence, inspire a lot of sub-1 V bandgap voltage reference circuit designs. Banba's architecture is the most common among industries as well as academic researchers. The following sections present modifications to this sub-1 V bandgap voltage reference circuit by resistive division, where the input voltage constraint to the opamp is lowered and, thus, overcomes the common-mode input voltage range problem to achieve sub-1 V opamp based bandgap voltage reference circuits.

\subsection{Divided resistive $V_{B E}-L e u n g$ and Mok's voltage reference: 2002}

Although the resistor factor $R_{4} / R_{3}$ can help to overcome the sub-1 V output voltage problem, such that the output voltage can be smaller than $1.2 \mathrm{~V}$, the bandgap voltage reference circuit in Figure 5 still cannot work with a sub-1 V supply voltage. The 


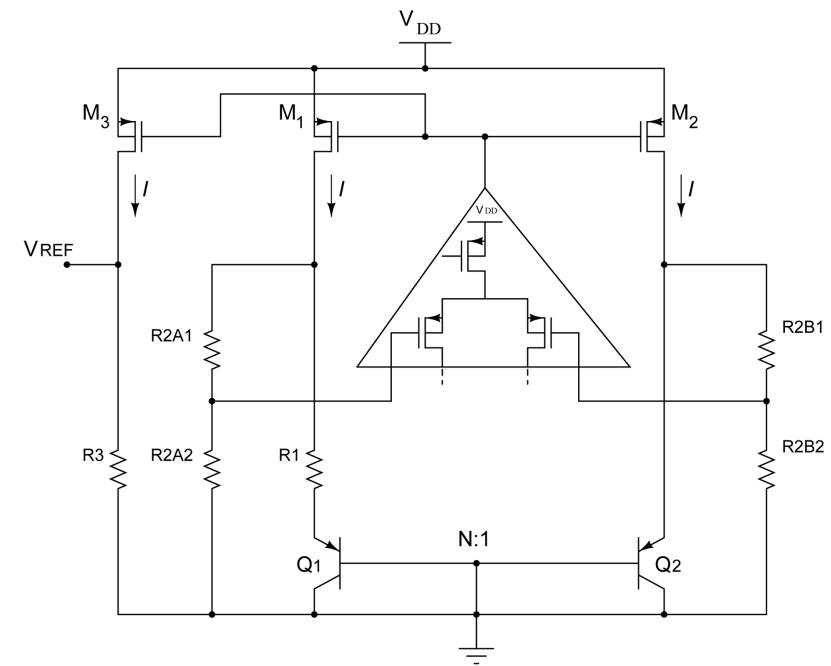

Figure 6. Leung and Mok voltage reference: 2002.

reference core circuitry proposed by Banba et al. [6] is modified by Leung and Mok [7]. The main differences are that an amplifier with a PMOS input stage is used and the inputs of the amplifier are connected to nodes $N_{1}$ and $N_{2}$ instead of $N_{3}$ and $N_{4}$. If a P-channel differential input stage opamp is used, the supply voltage is limited by the common-mode input voltage of the opamp. As a result, the input voltage to the opamp has to be low enough to make sure the P-channel input MOSFET pair are operating in the saturation region. Modified bandgap voltage reference circuit is shown in Figure 6 to lower the input voltages of the opamp by applying resistive voltage divider [7]. Assume $R_{1 A}=R_{2 A}$ and $R_{1 B}=R_{2 B}$. When the opamp has large gain, the inverted feedback loop of the amplifier will ensure $V_{A}=V_{B}$. As a result, reference voltage yields:

$$
V_{\mathrm{REF}}=\frac{R_{4}}{R_{1}}\left(V_{\mathrm{BE}_{1}}+\frac{R_{1}}{R_{3}} V_{T} \ln (N)\right) .
$$

The minimum operating supply voltage of this voltage reference circuit is given by Eq. (11) with $V_{\mathrm{BE}}$ being replaced by the resistive sub-divided $V_{\mathrm{BE}}$ as:

$$
V_{D D_{\min }}=\frac{R_{2_{B}}}{R_{2}} V_{\mathrm{BE}_{1}}+\left|V_{t h_{p}}\right|+2\left|V_{D S_{\mathrm{sat}}}\right|
$$

As a result, the minimum operating supply voltage of the voltage reference circuit is being lowered by a factor of $R_{2 B} / R_{2}$ acting on $V_{\mathrm{BE}, 1}$. We can almost conclude that the circuit has alleviated all the sub- $1 \mathrm{~V}$ voltage reference circuit design problems.

\subsection{Independent biased resistive divided $V_{B E}$ : Ker's voltage reference: 2002}

This circuit has a similar beta-multiplier circuit structure as the conventional opamp based bandgap voltage reference circuit where the current mirror transistors are directly connected to the temperature-sensitive BJTs [8]. As a result, when the temperature varies from $20^{\circ} \mathrm{C}$ to $100^{\circ} \mathrm{C}$, the $V_{\mathrm{BE}}$ voltage of BJT varies from $812.55 \mathrm{mV}$ to $646 \mathrm{mV}$, while the bandgap voltage reference circuit output stays almost the same. As a result, the drain to source voltages of $M_{1}, M_{2}$, and $M_{3}$ will not be the same and the transistors in the current mirror will suffer from the channellength modulation effect. Hence, the above difference in source to drain voltages will cause the output current differences in the current mirror formed by $M_{1}$, $M_{2}$, and $M_{3}$. However, such mirror output current mismatch problems exist in all source-to-drain voltage conditions when $V_{D D}$ is high. On the contrary, when $V_{D D}$ is low and close to $1 \mathrm{~V}, M$ will be working close to the linear region. As a result, the $I_{D S}$ current mismatch problem of the current mirror will be severe. Although the current mirror mismatch problem can be corrected by using a cascode current mirror, the extra layer of transistors in the cascode current mirror will increase the minimum operating supply voltage. Therefore, cascode current mirror is not applicable to correct the current mirror mismatch problem of the sub-1 V voltage reference circuit. Ker et al. [8] presented a modification to alleviate the current mirror mismatch problem by independently biasing the BJTs, instead of directly connecting the MOSFETs of the current mirror in the beta-multiplier circuit to the BJTs.

To alleviate the channel-length modulation effect that affects the performance of the current mirror, and hence the performance of the overall voltage reference circuit, Figure 7 shows a modified schematic, where the two BJTs are biased independently with two independent current sources instead of the opamp driven current sources. The independent biased resistive divided $V_{\mathrm{BE}}$ opamp based beta-multiplier bandgap sub$1 \mathrm{~V}$ voltage reference circuit was first proposed by Ker et al. [8].

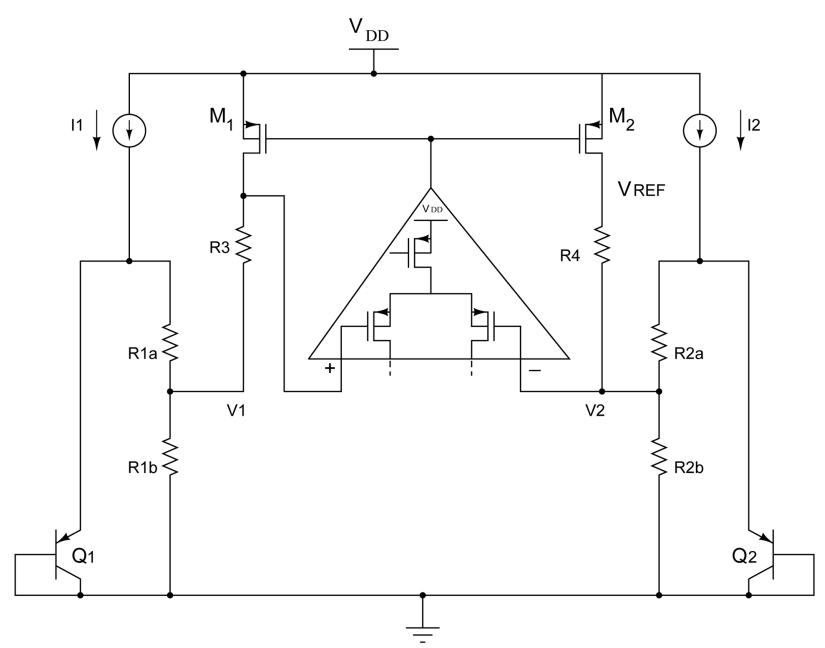

Figure 7. Ker's voltage reference: 2006. 
Similar to the Banba's voltage reference circuit, resistive sub-division is applied to reduce the input voltage to the opamp. When implementing the circuit, any current source that can operate with sub-1 $\mathrm{V} V_{D D}$ can be applied. Note that although the current generated by the Widlar current source is PTAT, applying it to the sub-1 V opamp based beta-multiplier bandgap voltage reference circuit by resistive sub-division with independent biased $V_{\mathrm{BE}}$ will not affect the performance of the system as long as $I_{1}=I_{2}$, and the two current sources have the same thermal property. The reference voltage is:

$$
\begin{aligned}
V_{\mathrm{REF}}= & {\left[\left(\frac{R_{1 B}}{R_{1 B}+R_{1 A}}+R_{4}\right) \frac{\Delta V_{\mathrm{BE}_{1,2}}}{R_{3}}+V_{\mathrm{BE}_{2}}\right] } \\
& \frac{R_{1 B}}{R_{1 B}+R_{1 A}} .
\end{aligned}
$$

Nevertheless, all resistive division voltage reference circuits require resistors with large resistance to scale down the reference voltage. The use of resistors with large resistance causes the resistive division voltage reference circuit to suffer from the problems of large silicon area. Use of dummy resistors for avoiding process variations results in additional area consumption. Area related problem is aggravated due to implementing resistor-matching techniques used of resistors, such as inter-digitization; which is area consuming. The production cost of this kind of bandgap voltage reference circuit is high, which prohibits their implementation in many existing applications.

\subsection{Annema's voltage reference: 2012}

Most of the sub-1 $\mathrm{V}$ bandgap voltage reference designs are based on the structure introduced by Banba et al. [6]. For low-power operation, high-ohmic resistors (occupying a large area) must be used in all these techniques, leading to an immediate trade-off between power consumption and chip-area, as we have discussed in the previous subsection. However, this trade-off prevents the local generation of reference voltages where they are required: either the power penalty or the area penalty would be too significant. Alternative topologies that do not require high-ohmic resistors typically are non-bandgap based reference circuits, relying on threshold voltages, and hence require trimming to achieve low spread.

This paper presents a sub-1 V bandgap voltage reference that circumvents the power-area trade-off of conventional sub-1 V bandgap voltage reference, aiming at local reference voltage generation wherever a reference voltage is needed in a die [9]. To break the power-area trade-off, no resistive averaging or subdivision can be used to get sub-1 V operation, while a drive to minimum area results in avoiding any topology using opamps, high ohmic resistors, or multiple diodes. The topology of the bandgap voltage reference by Annema et al. [9] is shown in Figure 8. The PMOS transistors $M_{P 1}$ and $M_{P 2}$ are biased in subthreshold region. $M_{P 1}$ is wider than $M_{P 2}$, which is the necessary condition for PTAT voltage generation if currents are equal in both branches. Along with this condition, Annema et al. have used shorter-length $M_{P 1}$ compared to $M_{P 2}$. By this technique, they have exploited reverse-short channel effects and yielded temperature-independent offset voltage $\Delta V_{t h}$.

$$
\begin{aligned}
V_{\mathrm{REF}} & =V_{T} \ln (A) N+V_{\mathrm{BE}}-N \Delta V_{t h} \\
& =V_{\mathrm{REF}_{\text {conven }}}-N \Delta V_{t h} .
\end{aligned}
$$
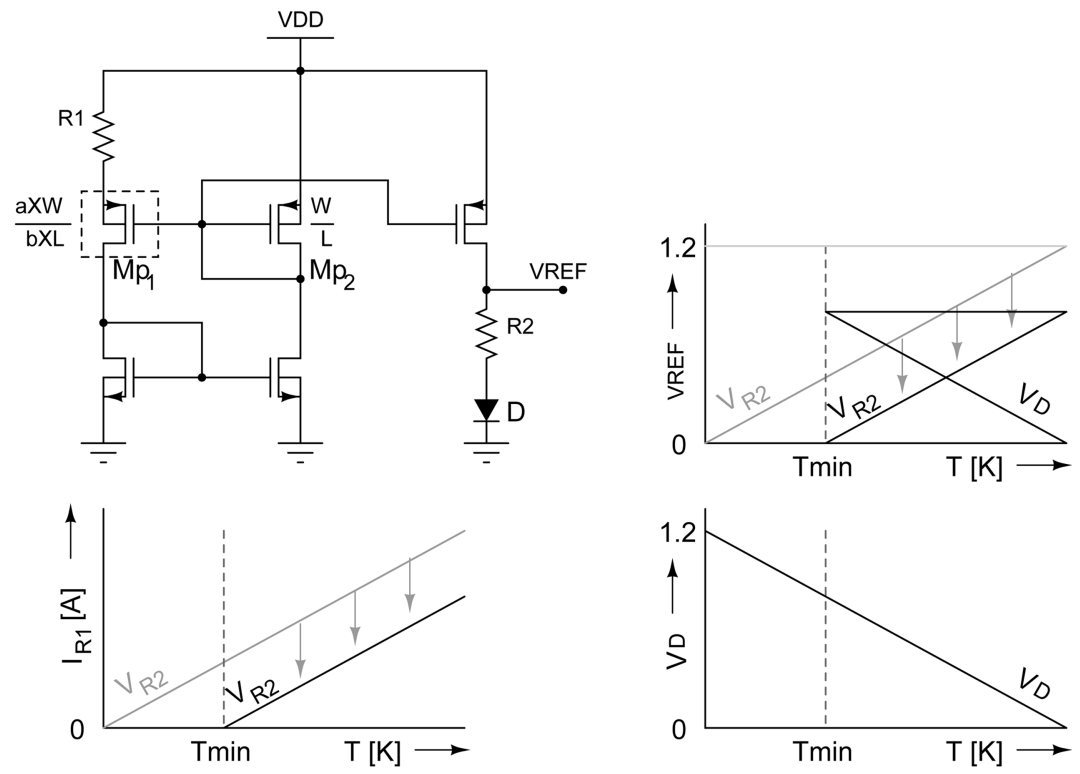

Figure 8. Principle of Annema's voltage reference: 2012. 
The proposed circuit operates at supply voltage of $1.1 \mathrm{~V}$ over temperature range of -45 to $135^{\circ} \mathrm{C}$ for the reference voltage value of $944 \mathrm{mV}$. The most attractive aspect of this circuit is it consumes only $1.4 \mu \mathrm{W}$ of power along with $0.0025 \mathrm{~mm}^{2}$ of silicon area and produces $30 \mathrm{ppm} /{ }^{\circ} \mathrm{C}$ of accuracy with lesser spread in reference voltage across process variations. Due to these unique low area and power consumption combination along with process tolerance quality, it coins a new concept as local generation of reference voltage.

\subsection{Najafizadeh's voltage reference using Filanosky's ZTC: 2004}

A conventional band-gap voltage reference is generally used since its output voltage is stable against temperature and process variations. Banba and other researchers have figured out the solutions for sub-1 V bandgap reference circuit, which also operates at below $1 \mathrm{~V}$. However, It was predicted in the ITRS road map that in year 2010, the mainstream power supply voltage would be around $0.6 \mathrm{~V}$. Therefore, to solve this issue, many researchers have moved towards MOS based voltage reference. MOS based voltage references are not very much process-friendly, but good in terms of power and area consumption. Among the many topologies of CMOS based voltage reference circuits, ZTC based reference design is a quite uncommon but innovative concept.

As in the study of Filanosky et al. [10], if MOSFET is biased at a specific gate-source voltage and drain current (i.e. bias point), the bias point does not change irrespective of change in temperature. It is called a ZTC point. Figure 9 shows the ZTC point. ZTC point arises due to the effect of mutual compensation of mobility and threshold voltage. This technique is implemented by Najafizadeh along with Filanosky in 2004 [11]. The presented voltage reference circuit is shown in Figure 10.

Low-voltage standard PTAT current generator is

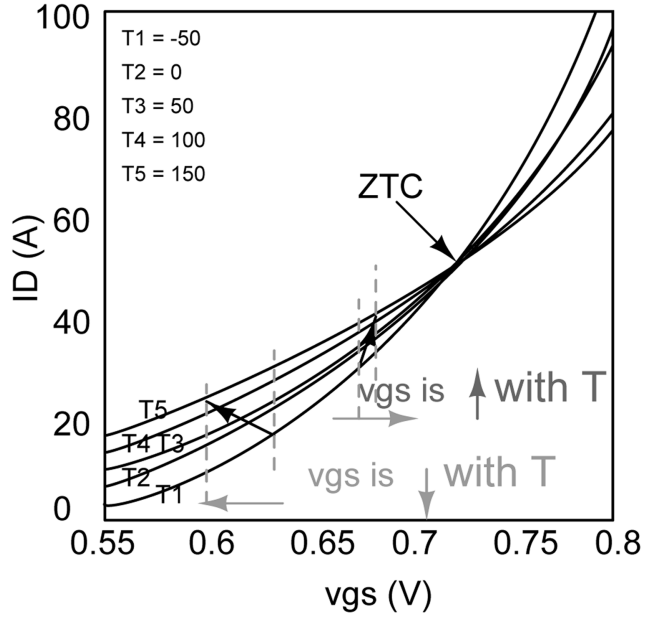

Figure 9. Filanosky's concept of ZTC: 2001.

implemented using $M_{1}-M_{4}$ and $R_{B}$. The reference core circuit consists two diode connected transistors $M_{7}$ and $M_{8}$ operating below the ZTC point, and two resistors, $R_{1}$ and $R_{2}$. Transistors $M_{5}$ and $M_{6}$ supply PTAT PTAT currents to the transistors $M_{7}$ and $M_{8}$. The output reference voltage is given by:

$$
V_{\mathrm{REF}}=\frac{V_{\mathrm{GS}_{7}}}{1+R_{1} / R_{2}}+\frac{V_{\mathrm{GS}_{8}}}{1+R_{1} / R_{2}} .
$$

4.7. Leung and Mok's voltage reference: 2003 In their paper, Leung and Mok presented a voltage reference based on weighted gate-source voltage difference between an NMOS and a PMOS [12]. The proposed CMOS voltage reference is shown in Figure 11 . The circuit has 3 sections:

i. A bias circuit $\left(M_{1}-M_{4}\right.$ and $\left.R_{B}\right)$;

ii. Start-up circuit $\left(M_{S 1}-M_{S 3}\right)$;

iii. Reference core $\left(M_{P}, M_{N}, R_{1}\right.$, and $\left.R_{2}\right)$.

Long channel lengths are selected for eliminating the channel modulation effect and biasing the transistors in saturation region at lower $V_{\mathrm{GS}}$ values.

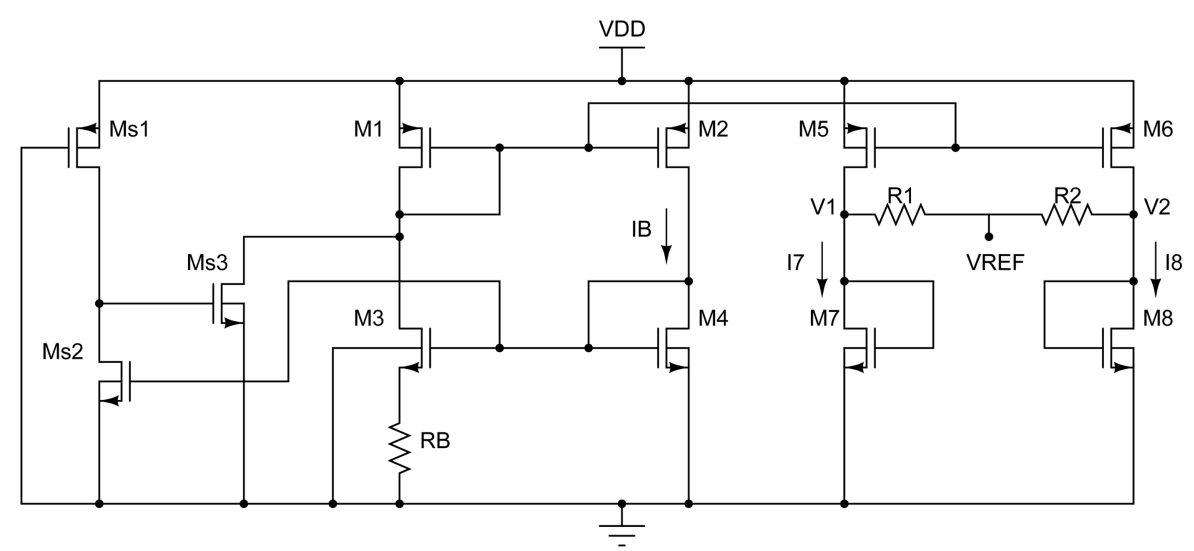

Figure 10. Najafizadeh voltage reference: 2004. 


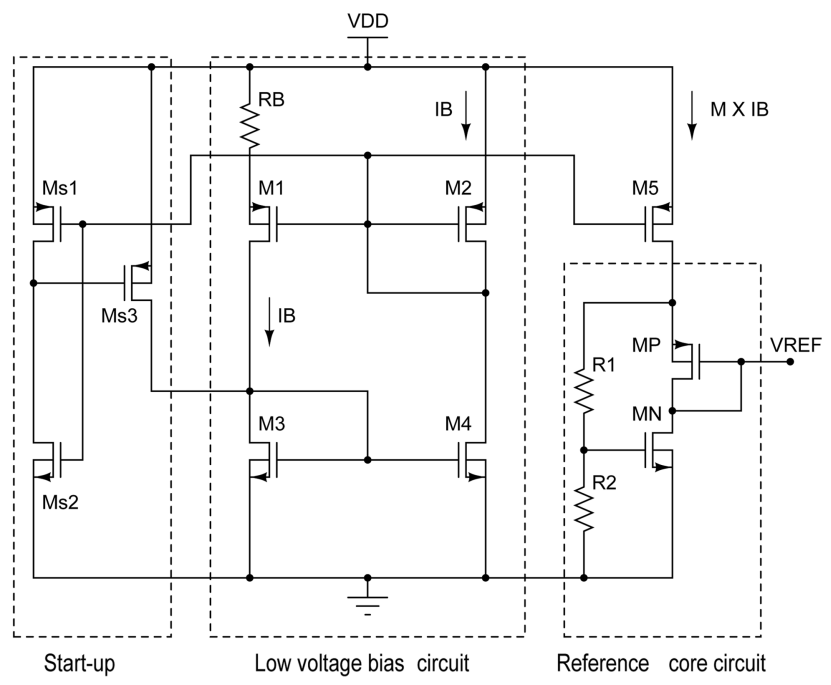

Figure 11. Leung and Mok's voltage reference: 2003.

Assume the current flowing through $R_{1}$ and $R_{2}$ is negligible; the bias current to $M_{P}$ and $M_{N}$ is $M$ times $I_{B}$. The reference voltage is given by:

$$
V_{\mathrm{REF}}=\left(1+\frac{R_{1}}{R_{2}}\right) V_{\mathrm{GS}_{n}}-V_{\mathrm{GS}_{p}} .
$$

A key factor in designing reference is keeping both $M_{P}$ and $M_{N}$ in deep saturation region. Therefore, the gatesource voltage is governed by the fundamental equation of MOS transistors in saturation region.

The minimum supply voltage should be considered at the minimum operable temperature as threshold voltages are at maximum. The minimum supply voltage $V_{D D_{\min }}$ is given by:

$$
V_{D D_{\text {min }}}=\left(1+\frac{R_{1}}{R_{2}}\right) V_{\mathrm{GS}_{n}}+\left|V_{D S_{5, \mathrm{sat}}}\right| .
$$

The minimum supply voltage is $1.4 \mathrm{~V}$ and it can be reduced if lower threshold voltage devices are used. Iannaccone and his research group have presented a series of articles from 2005 to 2014 .

These papers are the extended version of the topology, which was presented by Leung and Mok in 2003.

\section{Iannaccone's series of voltage references}

Iannaccone has significantly contributed to reference circuits. He has proposed few important topologies, which span almost over a decade, i.e. from 2005 to 2014. We have tried to study his work in this section.

\subsection{Reference voltage: CICC-2005}

In CICC'2005, Iannaccone and Vita presented a voltage reference, which could be implemented in any standard CMOS technology, based on the weighted gate-source voltage difference between two NMOS transistors [13].

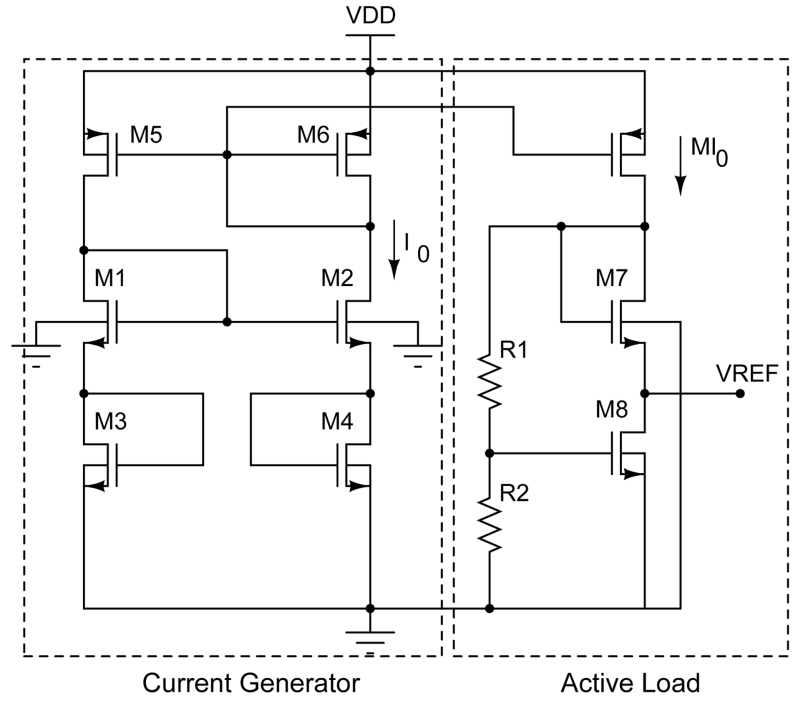

Figure 12. Vita-Iannaccone voltage reference: CICC-2005.

Such solution leads to a perfect cancellation of the effect of the temperature dependence of carrier mobility, which was a drawback in Leung and Mok's topology.

The presented voltage reference generator is shown in Figure 12. It has a supply-independent current generator, $I_{0}$; such current is then amplified and injected into an active load to generate the reference voltage. A temperature-invariant voltage reference is obtained by compensating the temperature dependence of the generated current with the temperature dependence of the NMOS threshold voltage. The final reference voltage is:

$$
V_{\mathrm{REF}}=\left(1+\frac{R_{1}}{R_{2}}\right) V_{\mathrm{GS}_{8}}-V_{\mathrm{GS}_{7}} .
$$

The minimum supply voltage limitation is imposed by the $V_{D D}$ independent current generator circuit. The minimum allowable supply voltage is:

$$
V_{D D_{\text {min }}}=\left|V_{\mathrm{GS}_{6}}\right|+V_{D S_{2, \min }}+V_{\mathrm{GS}_{4}} \text {. }
$$

The supply voltage must be larger then $1.5 \mathrm{~V}$ for the used AMS $0.35 \mu \mathrm{m}$ CMOS process.

Unfortunately, in this design, the temperature coefficient degrades due to non-ideal effects, i.e. channel length modulation and body effect. An experimental temperature coefficient of $25 \mathrm{ppm} /{ }^{\circ} \mathrm{C}$ is achieved.

\subsection{Reference voltage: VLSI Symposium-2006}

As the battery-operated systems have gained popularity after 2005, the reference circuit must consume low power and area with reasonable accuracy. The series of De Vita and Innaccone [13] consumes $2.25 \mu \mathrm{W}$ of power at the supply voltage of $1.5 \mathrm{~V}$ with the consumption of $0.08 \mathrm{~mm}^{2}$ chip area. The major reason behind this performance is power and area trade-off due to presence 


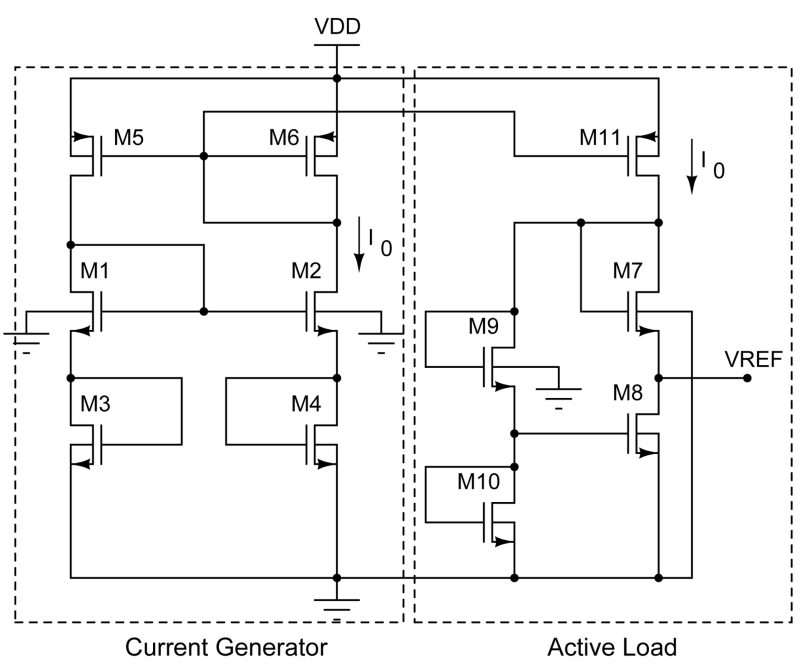

Figure 13. Vita-Iannaccone voltage reference: VLSI Symposium-2006.

of resistors. Therefore, De Vita et al. [14] have replaced the resistors with MOSFETs to break the power and area trade-off. The enhanced reference circuit is given in Figure 13.

Similar to De Vita and Iannaccone [13], the active load is used to generate the reference voltage in this study. It consists of two NMOS transistors, $M_{7}$ and $M_{8}$, biased by $I_{0}$, and a voltage divider formed by $M_{9}$ and $M_{10}$. All transistors in the active load operate in the saturation region. The final reference voltage is:

$$
V_{\mathrm{REF}}=V_{t h}+\left[\frac{1}{\sqrt{k_{8}}}\left(1+\sqrt{\frac{W_{10} / L_{10}}{W_{9} / L_{9}}}\right)-\frac{1}{\sqrt{k_{7}}}\right] \sqrt{2 I_{0}}
$$

where, $k=\mu \operatorname{Cox} W / L$ and $I_{0}$ is supply independent current.

This reference circuit consumes $0.12 \mu \mathrm{W}$ of power at the supply voltage of $1.5 \mathrm{~V}$ with the consumption of $0.015 \mathrm{~mm}^{2}$ chip area. The improvements are 5 and 18 times with regards to power and area, compared to the topology of De Vita and Iannaccone [13]. Moreover, this design provides both a complete suppression of the temperature dependence of mobility and a compensation of non-ideal effects, i.e. channel length modulation and body effect. Hence, temperature insensitivity is also enhanced twice, i.e. $\approx 12 \mathrm{ppm} /{ }^{\circ} \mathrm{C}$. Unfortunately, the minimum supply voltage is still quite high, that is, larger than $1.5 \mathrm{~V}$, and thus it is not suitable for low-voltage applications.

\subsection{Reference voltage: JSSC-2007}

In their paper, De Vita and Iannaccone [15] presented a CMOS voltage reference with a minimum supply voltage smaller than $1 \mathrm{~V}$, really enabling low-voltage operation, which still provides a very good temperature coefficient. Let us consider the active load of the proposed reference voltage generator, shown in Figure 14.

Assuming that all transistors of the active load work in the saturation region, the output reference voltage would be given by:

$$
V_{\mathrm{REF}}=V_{t h_{10}}+\frac{\eta V_{T}}{N-1} \sqrt{\frac{W_{4} / L_{4}}{W_{10} / L_{10}}} \ln \left(\frac{W_{3} / L_{3}}{W_{1} / L_{1}}\right)
$$

The minimum supply voltage is imposed by the current generator circuit. It is given as:

$$
V_{D D_{\min }}=\left|V_{\mathrm{GS}_{5}}\right|+V_{D S_{1, \min }} .
$$

The minimum supply voltage is $0.9 \mathrm{~V}$ in the AMS $0.35 \mu \mathrm{m}$ CMOS process. The accuracy achieved in this architecture is $10 \mathrm{ppm} /{ }^{\circ} \mathrm{C}$ along with improvement in power consumption. Power consumption is limited to only $0.0036 \mu \mathrm{W}$.

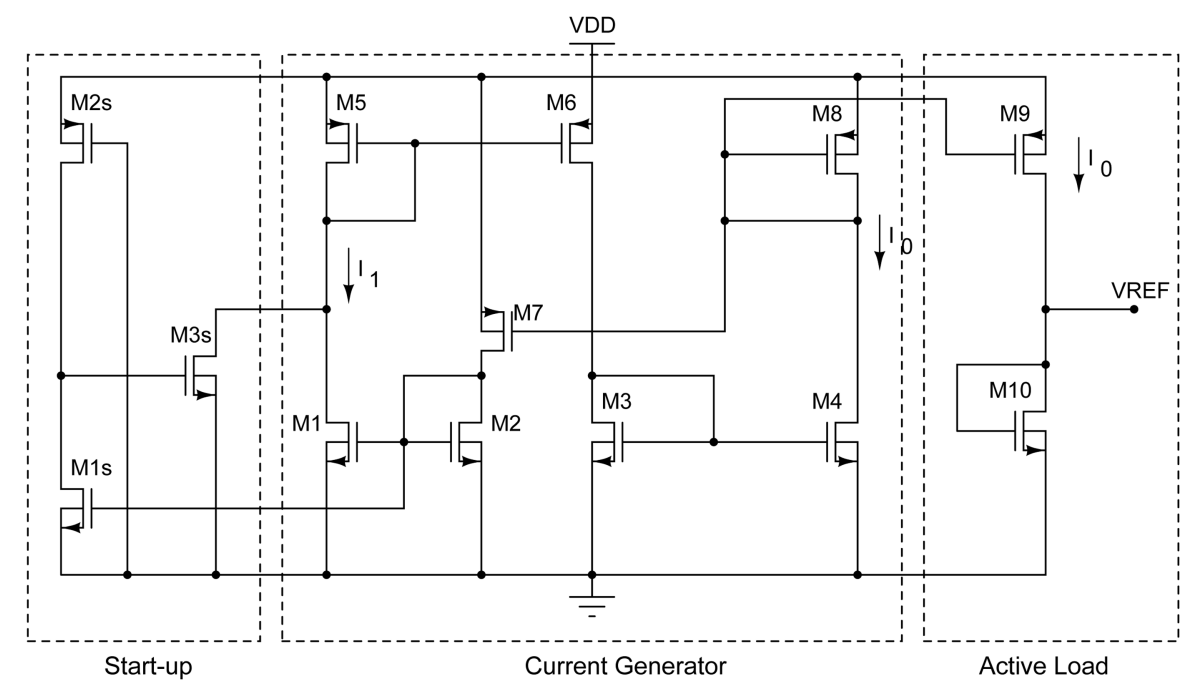

Figure 14. Vita-Iannaccone voltage reference: JSSC-2007. 


\subsection{Reference voltage: JSSC'2011 and journal of circuit theory and applications, i.e. JCTA'14}

An ultra-low voltage and ultra-low power voltage reference circuit was presented by Magnelli et al. in JSSC'2011 [16]. They fabricated the circuit in the UMC $180 \mathrm{~nm}$ CMOS process. Importantly, the circuit works with all transistors in subthreshold region, thus allowing a drastic reduction of minimum supply voltage and power consumption. The proposed solution represents a significant advance in low-power, lowvoltage reference circuit design, which chooses a new milestone for extremely low power operation. The power consumed by the circuit is only $2.6 \mathrm{nW}$, which is about one order of magnitude lower than that of the best results found in the literature at the time, and the minimum supply voltage for correct operation falls to $0.45 \mathrm{~V}$. However, across the process, the circuit is a little vulnerable; thus, external trimming is required to achieve the specified accuracy and DC voltage level.

Even after achieving power dissipation down to $2.6 \mathrm{nW}$, Iannaccone and his group were working aggressively on reduction of power dissipation. Iannaccone et al. [17] presented a voltage reference consisting of only two nMOS transistors with different threshold voltages. Measurements performed on 23 samples from a single batch show a mean reference voltage of $275.4 \mathrm{mV}$. The subthreshold conduction and the low number of transistors enable us to achieve a mean power consumption of only $40 \mathrm{pW}$. The minimum supply voltage is $0.45 \mathrm{~V}$, which is considered with one of the ultra-low power reference topologies. The mean $\mathrm{TC}$ in the temperature range of 0 to $120^{\circ} \mathrm{C}$ is $105.4 \mathrm{ppm} /{ }^{\circ} \mathrm{C}$. The occupied area is $0.018 \mathrm{~mm}^{2}$. The power supply rejection rate without any filtering capacitor is $-48 \mathrm{~dB}$ at $20 \mathrm{~Hz}$ and $-29.2 \mathrm{~dB}$ at $10 \mathrm{kHz}$.

\section{Voltage reference circuits based on subthreshold MOSFETs}

During 1995 to 2000, the demand for analog integrated circuits that had to operate under lower supply voltages and ultra-low power rapidly increased, especially in applications like portable equipment (e.g., watches, handheld gadgets, smart phones, etc.). Fortunately, for the analog engineer, the sub-threshold voltage characteristics of MOSFET devices mimic the characteristics of Bipolar devices. Therefore, subthreshold-based MOSFET was an potential replacement for BJTs. Therefore, the nanopower regime opened up a wide perspective for reference circuits using a subthreshold transistor. Even though these transistors are quite dependent on process, they have become famous due to their ultra-low power and low area consumption capabilities. We will see some reference circuits based on subthreshold biasing principle.

\subsection{Ytterdal's voltage reference: 2003}

As discussed in Section 4.6, Najafizadeh and Filanovsky [11] have designed MOSFET-based reference circuit to solve the problem of supply voltage reduction due to shrinking technology. Ytterdal [18] also solved a similar supply-related problem using MOS devices; however, he used subthreshold biased MOS transistors for replacing BJTs, where Najafizadeh used a concept of ZTC.

Ytterdal basically used the technique presented by Banba. However, he replaced bipolar devices with subthreshold-based MOSFETs. As a consequence, Ytterdal was able to reduce the supply voltage well below $1 \mathrm{~V}$. He also used low-threshold voltage NMOS transistors to reduce $V_{D D_{m i n}}$. Moreover, he also reduced the threshold voltages of PMOS by bulkbiasing. The proposed topology is given in Figure 15.

Due to these techniques, the reference voltage circuits operate at $0.6 \mathrm{~V}$ for $0.13 \mu \mathrm{m}$ digital CMOS

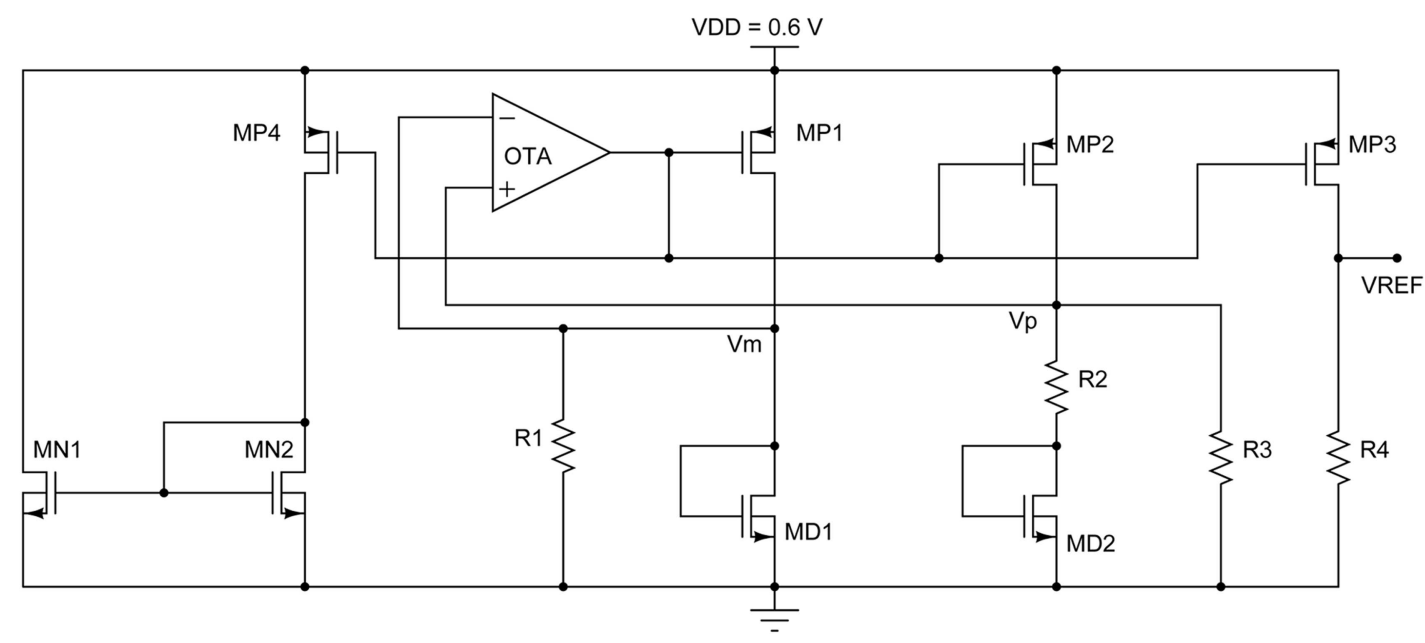

Figure 15. Ytterdal voltage reference: 2003. 
technology. The reported temperature coefficient is $93 \mathrm{ppm} /{ }^{\circ} \mathrm{C}$.

\subsection{Reference voltages by Giustolisi et al., 2003, and Huang et al., 2006}

Similar to Ytterdal, Giustolisi et al. [19] have also used identical principle to develop a reference circuit. They exploit the fact that the gate-source voltage decreases linearly with temperature, if it is biased with a constant drain current.

As shown in a Figure 16, the current $I_{R_{1}}$ is the minimum supply voltage imposed by the current generator circuit. It is given as:

$$
I_{R_{1}}=\frac{V_{\mathrm{GS}_{1}}}{R_{1}} .
$$

This current is then provided for the second subcircuits $M_{5}$ and $M_{6}$ and the output voltage is given by:

$$
V_{\mathrm{REF}}=K_{1} V_{\mathrm{GS}}+K_{1} V_{T}
$$

where $K_{1}$ and $K_{2}$ are the combinations of transistor aspect ratios and resistor ratios. After selection of proper values for resisters and MOSFETs, the proposed architecture achieves an output voltage of $0.2953 \pm 0.0108 \mathrm{~V}$ and a temperature coefficient of $119 \pm 35.7 \mathrm{ppm} /{ }^{\circ} \mathrm{C}$ for the temperature range of -25 to $125^{\circ} \mathrm{C}$. Operable minimum supply voltage is $1.2 \mathrm{~V}$ with line regulation of $2 \mathrm{mV} / \mathrm{V}$. The current consumption is $3.6 \mu \mathrm{A}$.

The circuit presented by Giustolisi et al. is complex and consumes larger area and power compared to the reference circuit proposed by Huang et al. [20]. The circuit by Huang et al. is given in Figure 17 . The comparison is done on the basis of identical working principle, i.e. gate-source voltage compensated by thermal voltage (core transistors are operating in subthreshold region). The presented circuit by Huang

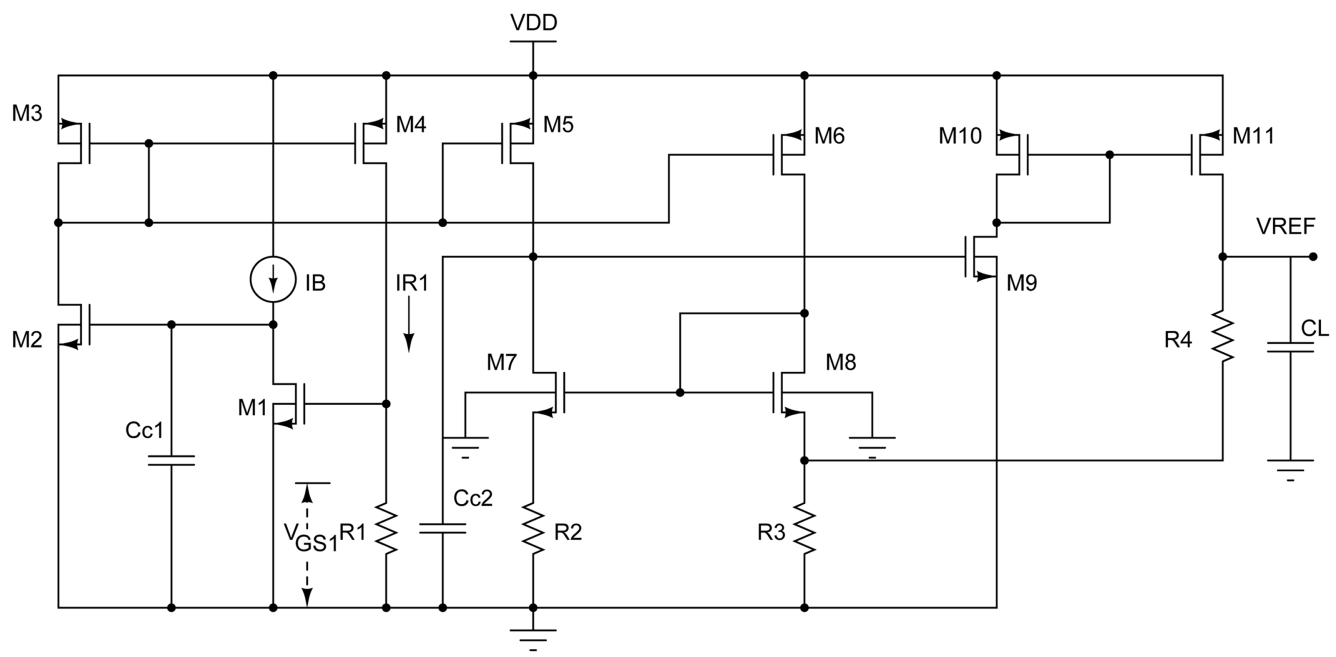

Figure 16. Giustolisi et al.: voltage reference, 2003.

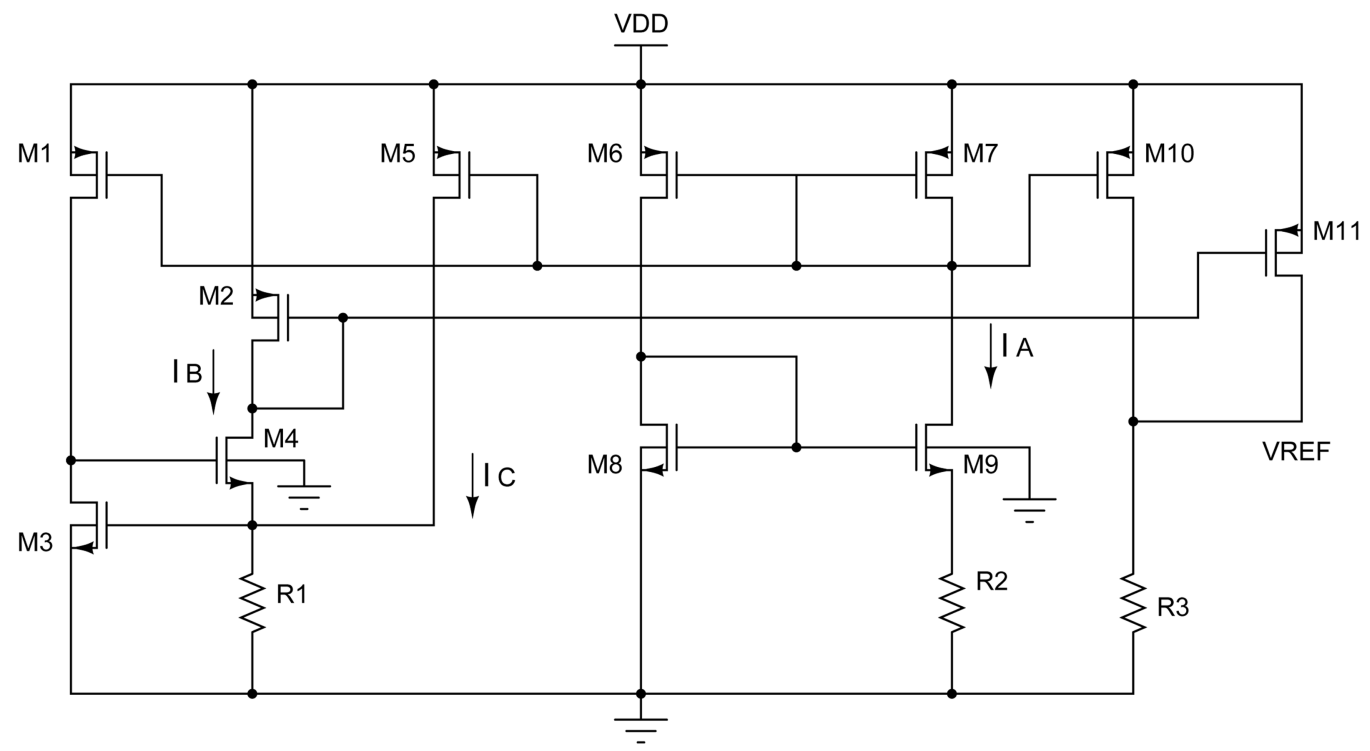

Figure 17. Huang et al.: voltage reference 2006. 
et al. is a less complicated low-voltage, low-power CMOS reference circuit using subthreshold biasing concept.

They have also compensated the channel-length modulation effect to improve the performance. The mean reference voltage is $\approx 222 \mathrm{mV}$ with line regulation of $2 \mathrm{mV} / \mathrm{V}$ (for $V_{D D}$ varies from 0.9 to $2.5 \mathrm{~V}$ ). Peak-to-peak variation of $6 \mathrm{mV}$ is reported for temperature from $-20^{\circ} \mathrm{C}$ to $120^{\circ} \mathrm{C}$. The chip area is less than $0.0238 \mathrm{~mm}^{2}$ with minimum power consumption of $3.3 \mu \mathrm{W}$.

\section{High-order curvature correction}

Precision bandgap references are significantly necessary for many applications ranging from solely analog, to mixed-mode and purely digital circuits, such as data converters, digital data RAMS, power converters, and memory controlling circuits. They are known for their high accuracy and temperature-independent behavior. The reference voltage is required to be stable over the supply voltage and temperature variations. It should also be implemented without any modification of the standard fabrication process.

A conventional bandgap reference circuit is firstorder temperature compensated. Basically, it is an appropriately scaled sum of negative TC voltage $V_{\mathrm{BE}}$ and positive TC voltage $V_{T}$, which is the thermal voltage. With regard to nonlinearity of the voltage $V_{\mathrm{BE}}$, TC of first-order temperature compensated references is always limited between 20 and $100 \mathrm{ppm} /{ }^{\circ} \mathrm{C}$. Therefore, to overcome this limitation, many highorder temperature compensation approaches have been presented. Compensation of the high-order temperature dependent terms of the reference voltage (nonlinear terms) is therefore referred to as curvature correction or curvature compensation. The primary curvature correction techniques used in practice are, for example, the quadratic temperature compensation proposed by Song and Gray [21], the exponential temperature compensation developed by Lee et al. [22], the piecewise linear curvature correction presented by Rincon-Mora and Allen [23], and the temperature dependent resistor ratio with a high resistive poly resistor and a diffusion resistor proposed by Leung et al. [24]. The accuracy of bandgap references has been enhanced with these techniques; however, they also increase the requirements of precise matching of the current mirror. Some of the popular curvature correction techniques will be discussed in the next subsections.

\subsection{Song's voltage reference: 1983}

The nonlinear relationship between $V_{\mathrm{BE}}(T)$ and temperature is expressed as:

$$
\begin{aligned}
V_{\mathrm{BE}}(T)= & V_{\mathrm{BG}}-\left[V_{\mathrm{BG}}-V_{\mathrm{BE}}\left(T_{r}\right)\right] \frac{T}{T_{r}} \\
& -(\eta-m) V_{T} \ln \left(\frac{T}{T_{r}}\right),
\end{aligned}
$$

where $V_{\mathrm{BG}}$ is the bandgap voltage of $\mathrm{Si}$ as a function of temperature, $T_{r}$ is the reference temperature, $\eta$ is a temperature constant dependent on technology, and $m$ is the order of the temperature dependence of the collector current. The third term of Eq. (24) is proportional to $\mathrm{PTAT}^{2}$ voltage.

Song et al. proposed to add a $\mathrm{PTAT}^{2}$ term to the linear PTAT term to compensate for the junction curvature. The concept is clearly demonstrated by Song et al. The optimum values of the PTAT $^{2}$ correction voltage, the first-order corrected $V_{\mathrm{REF}}$, and second-order corrected $V_{\mathrm{REF}}$ at $25^{\circ} \mathrm{C}$ were measured and recorded $61 \mathrm{mV}, 1.256 \mathrm{~V}$ and $1.192 \mathrm{~V}$, respectively.

\subsection{BJT current subtraction - Gunawan's voltage reference: 1993}

As discussed by Gunawan et al. [25], a current source with second-order temperature dependency can be conveniently obtained by the BJT in the same way as that in the output stage of the opamp based betamultiplier bandgap voltage reference circuit. (The second-order temperature dependent nature of the BJT $V_{\mathrm{BE}}$ voltage is one of the major reasons for the need to implement second-order temperature compensation to obtain a low TC reference voltage over a wide temperature range.) The presented circuit is shown in Figure 18. When this BJT current source is appropriately connected to the voltage reference circuit, it forms a current sink and, hence, functions as secondorder current subtraction circuit to achieve secondorder curvature correction. Consider the schematic of Banba's opamp based bandgap voltage reference circuit with the BJT current subtraction. This topology is an extension of Banba's concept. The resistors in the

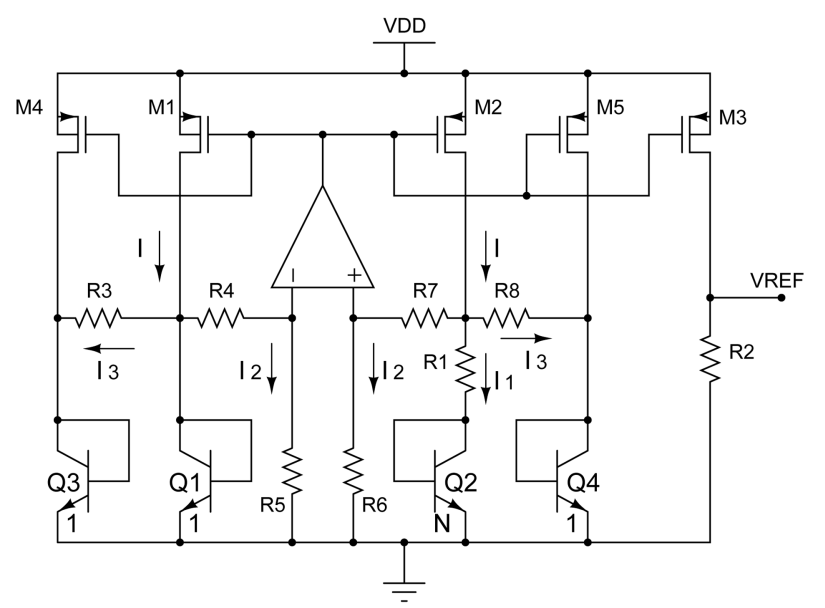

Figure 18. Gunwan's voltage reference topology: 1993. 
circuit satisfy $R_{3}=R_{8}, R_{4}=R_{7}$, and $R_{5}=R_{6}$. The emitter areas of $Q_{1}, Q_{2}, Q_{3}$, and $Q_{4}$ are in the ratio of $1: N: 1: 1$. Note that the negative feedback configured opamp will bias the input voltages to be the same; therefore, we write:

$$
V_{A}=V_{B}
$$

Therefore, with $R_{5}=R_{6}$, the currents that flow through $R_{5}$ and $R_{6}$ will be the same and equal to $I_{2}$. Assume the opamp is ideal; no current will be flowing in/out of $V+$ and $V$. As a result, the currents that flow through $R_{4}$ and $R_{7}$ will be the same; hence:

$$
I_{1}=\frac{V_{T} \ln (N)}{R_{1}}
$$

and:

$$
I_{2}=\frac{V_{\mathrm{BE}_{1}}}{R_{4}+R_{5}}
$$

and:

$$
I_{3}=\frac{V_{T} \ln \left(T / T_{\mathrm{nom}}\right)}{R_{3}} .
$$

The KCL at node $\mathrm{C}$ implies $I=I_{1}+I_{2}+I_{3}$, and the current mirror pair formed by $M_{2}$ and $M_{3}$ copies the current to the output branch. By selecting $S_{2}=S_{3}$, we shall obtain:

$$
V_{\mathrm{REF}}=K_{1}\left(V_{\mathrm{REF}_{\text {conven }}}+K_{3} \ln \left(T / T_{\text {nom }}\right) V_{T}\right),
$$

where:

$$
K_{1}=R_{2} /\left(R_{4}+R_{5}\right), \quad K_{2}=\left(R_{4}+R_{5}\right) / R_{1},
$$

and:

$$
K_{3}=\left(R_{4}+R_{5}\right) / R_{3} .
$$

Furthermore, it is clear that when $T=T_{\text {nom }}$, the voltage reference obtained by Eq. (29) will have the same form as that of the conventional bandgap voltage reference circuit at $T_{\text {nom }}$ (as studied in the previous sections).

At other temperatures, the high-order temperature dependent term $K_{3} \ln \left(T / T_{\text {nom }}\right) V T$ will compensate the second-order temperature dependent term of $V_{\mathrm{BE}, 1}$. This is achieved by appropriate selection of the resistor $R_{3}$ to drive $Q_{3}$.

\subsection{Piecewise curvature correction - by Rincon-Mora and Phillip E. Allen: 1998}

Around the year 1998 and onwards, the demand for low voltage references appeared, especially in mobile battery-operated products such as cellular phones, pagers, camera recorders, and laptops. Consequently, low-voltage and low-quiescent current flows are intrinsic and required characteristics for increased battery efficiency. Along with that, financial considerations also require these circuits to be realized in relatively simple processes, such as standard CMOS. Owing to this requirements, Rincon-Mora and Phillip E. Allen have designed and fabricated a low-voltage, micropower, curvature-corrected bandgap circuit in a relatively inexpensive process, MOSIS CMOS $2 \mu \mathrm{m}$ N-well technology with an added P-base layer [23]. The P-base layer is used to create NPN transistors. However, a vanilla CMOS version of the circuit can also be designed by using lateral PNP transistors. The circuit implements a novel current-mode piecewiselinear curvature-correction technique.

Piecewise-linear curvature-correction is a novel concept implemented by Rincon-Mora and Phillip E. Allen. Afterwards, many researchers have used enhanced techniques based on this concept. Operation principle is given in Figure 19(a) and (b).

The curvature correction is based on the addition of a nonlinear component to the output of a first-order bandgap reference. This is used to offset the nonlinear behavior of $V_{\mathrm{BE}}$ with respect to temperature. The nonlinear component, in this case, is realized by $I_{\mathrm{NL}}$ in the current-mode topology of the circuit described in Figure 19(a). It is basically a current-mode piecewiselinear form of compensation. The essence of the circuit centers on current subtraction and the characteristics of non-ideal transistors. Figure 19(b) graphically illustrates the operation of the circuit throughout the temperature range. The $I_{\mathrm{NL}}$ behavior is described by:

$$
I_{\mathrm{NL}}=0 \quad \text { if, } \quad I_{V_{\mathrm{BE}}}>I_{\mathrm{PTAT}},
$$

or:

$$
I_{\mathrm{NL}}=K_{1} I_{\mathrm{PTAT}}-K_{2} V_{\mathrm{BE}} \quad \text { if, } \quad I_{V_{\mathrm{BE}}}<I_{\mathrm{PTAT}},
$$

where $K_{1}$ and $K_{2}$ are constants and defined by current mirroring ratios.

$$
\begin{aligned}
V_{\mathrm{REF}} & =K_{1}\left(K_{2}+V_{\mathrm{BE}_{1}}+K_{3} \ln \left(T / T_{\text {nom }}\right) V_{T}\right) \\
& =K_{1}\left(V_{\mathrm{REF}_{\text {conven }}}+K_{3} \ln \left(T / T_{\text {nom }}\right) V_{T}\right),
\end{aligned}
$$

where $K_{3}$ is multiplication constant.

Finally, the reference voltage $\left(V_{\mathrm{REF}}\right)$ is temperature compensated to exhibit a behavior that is graphically described by Figure 19(c) and (d). The lower temperature range is essentially a first-order bandgap, since the nonlinear component $\left(I_{\mathrm{NL}}\right)$ is zero. At higher temperatures, the resulting behavior is similar to that of the lower temperatures, but the operation is not. The nonlinear behavior of $I_{\mathrm{NL}}$ attempts to cancel non-linearity of $I_{V_{\mathrm{BE}}}$. Therefore, addition of $A I_{V_{\mathrm{BE}}}$, $B I_{\mathrm{PTAT}}$, and $C I_{\mathrm{NL}}$ gives the curvature correction operation as shown in Figure 19(d). The measured results show that the achieved accuracy is less than $20 \mathrm{ppm} /{ }^{\circ} \mathrm{C}$ and minimum operable supply voltage of $1.1 \mathrm{~V}$. 


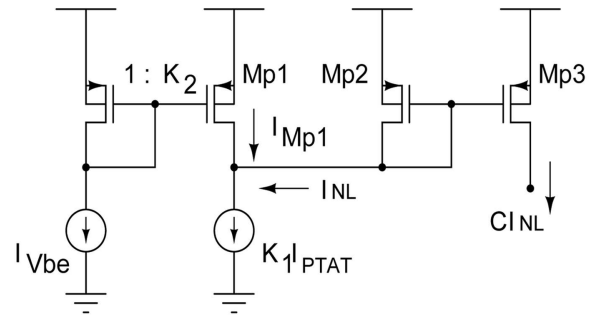

(a)

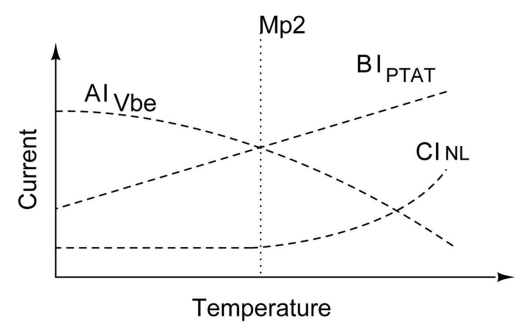

(c)

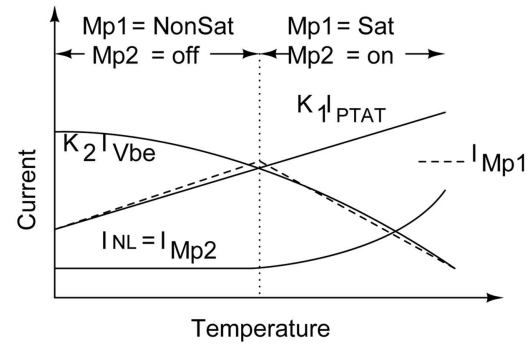

(b)

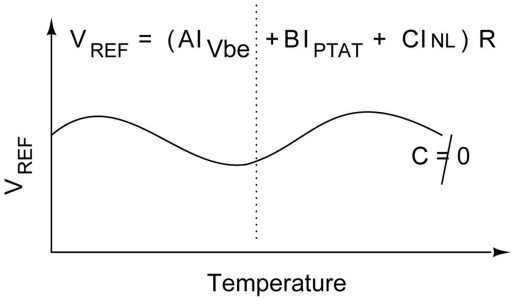

(d)

Figure 19. (a) and (b) Generation of the nonlinear current component. (c) and (d) Temperature dependence of the curvature-corrected bandgap reference.

\subsection{Curvature correction - by Malcovati's: 2001}

The circuit proposed by Malcovati et al. [26] is shown in Figure 20. It is one of the classic voltage references for curvature correction. Different values of $V_{\mathrm{BE}}$ can be obtained by different temperature-dependent collector currents. In this circuit, $Q_{2}$ is biased by a PTAT current:

$$
\begin{aligned}
V_{\mathrm{BE}_{2}}= & V_{\mathrm{BG}}\left(T_{r}\right)+\frac{T}{T_{r}}\left[V_{\mathrm{BE}}\left(T_{r}\right)-V_{\mathrm{BG}}\left(T_{r}\right)\right] \\
& +(\eta-1) \frac{k T}{q} \ln \left(\frac{T_{r}}{T}\right),
\end{aligned}
$$

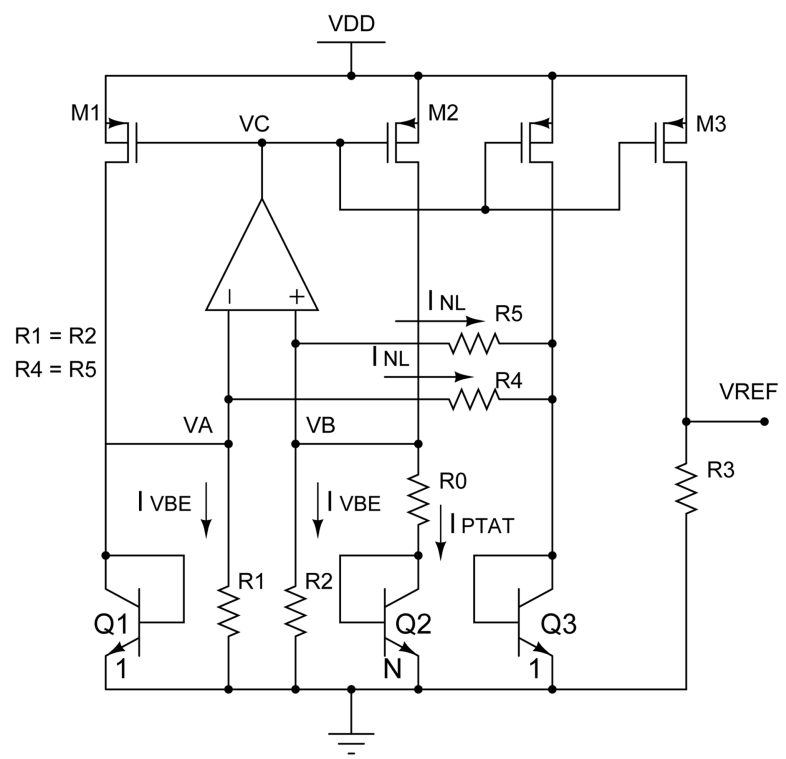

Figure 20. Schematic of the proposed bandgap circuit with curvature compensation by Malcovati. while $Q_{3}$ is biased by a temperature-independent current and $V_{\mathrm{BG}}$ is bandgap voltage of silicon:

$$
\begin{aligned}
V_{\mathrm{BE}_{3}}= & V_{\mathrm{BG}}\left(T_{r}\right)+\frac{T}{T_{r}}\left[V_{\mathrm{BE}}\left(T_{r}\right)-V_{\mathrm{BG}}\left(T_{r}\right)\right] \\
& +\eta \frac{k T}{q} \ln \left(\frac{T_{r}}{T}\right) .
\end{aligned}
$$

A non-linear current $I_{\mathrm{NL}}$, which is the current flowing through $R_{4}$, is generated and given by:

$$
I_{\mathrm{NL}}=\frac{V_{\mathrm{BE}_{2}}-V_{\mathrm{BE}_{3}}}{R_{4}}=\frac{V_{T}}{R_{4}} \ln \left(\frac{T}{T_{r}}\right) .
$$

Therefore, $V_{\mathrm{REF}}$ is given by:

$$
\begin{aligned}
V_{\mathrm{REF}} & =\left(I_{1}+I_{2}+I_{3}\right) R_{3} \\
& =\frac{R_{3}}{R_{1}} V_{T} \ln (N)+\frac{R_{3}}{R_{2}} V_{\mathrm{BE}_{2}}-\frac{R_{3}}{R_{4}} V_{T} \ln \left(\frac{T_{r}}{T}\right) .
\end{aligned}
$$

When the easy-control resistor ratio $R_{2} / R_{4}=\eta-1$, the non-linear voltage in $V_{B E, 2}$ is canceled. A theoretical zero TC $V_{\mathrm{REF}}$ can be obtained. However, it cannot always be achieved due to the non-ideal PTAT and temperature-independent currents from the temperature dependence of resistors. In addition, errors due to mismatch of current mirrors worsen the accuracy.

The proposed bandgap reference circuit by Malcovati et al. was fabricated in a $0.8 \mu \mathrm{m}$ Bi-CMOS technology. It achieved a temperature coefficient of $7.5 \mathrm{ppm} /{ }^{\circ} \mathrm{C}$. The line regulation was $212 \mathrm{ppm} / \mathrm{V}$, with a power consumption of only $92 \mu \mathrm{W}$ at room temperature. 


\subsection{Brief discussion of curvature correction techniques after 2001}

In 2003, Leung et al. [24] presented a low-voltage CMOS high-order curvature compensated bandgap reference based on a temperature-dependent resistor ratio. The proposed bandgap reference utilizes the negative temperature-coefficient high-resistive poly resistor in the CMOS process and the positive temperature- coefficient diffusion resistor to implement a temperaturedependent resistor ratio. This resistor ratio can effectively reduce the temperature drift of the bandgap reference voltage. The achieved temperature coefficient is $5.3 \mathrm{ppm} /{ }^{\circ} \mathrm{C}$ at a $2 \mathrm{~V}$ supply voltage, which consumes $23 \mu \mathrm{A}$ current. In 2005, Mitrea et al. [27] proposed a curvature-correction technique based on compensation of the base-emitter voltage nonlinearity, using it to correct the drain current of a MOS transistor working in weak inversion. Cancellation of the first- and secondorder terms of the polynomial expansion of the baseemitter voltage allows the reduction of the temperature coefficient of the bandgap reference. The measured temperature coefficient is about $10.5 \mathrm{ppm} /{ }^{\circ} \mathrm{C}$ over a temperature range of $10^{\circ} \mathrm{C}$ to $90^{\circ} \mathrm{C}$, while power consumption is subtly at higher side, i.e. $\approx 1.4 \mathrm{~mW}$. In 2006, Ker and Chen [28] proposed a curvaturecompensation technique, which had two output reference currents. These currents were generated by two current-mode bandgap references (the similar architecture to that of Leung and Mok in 2002 [7]). Both currents acted with concave and convex curves. Addition of these curves gave curvature compensated performance. The experimental results showed that the minimum operable supply voltage was $0.9 \mathrm{~V}$ and the output reference voltage was $536.7 \mathrm{mV}$ with a temperature coefficient of $19.55 \mathrm{ppm} /{ }^{\circ} \mathrm{C}$ from $0^{\circ} \mathrm{C}$ to $100^{\circ} \mathrm{C}$. In 2010, Guan et al. [29] presented an advanced version of Malcovati's design. They used current-mode curvature compensation technique. The circuit delivered an output voltage of $1.09 \mathrm{~V}$ and achieved the lowest reported temperature coefficient of $3.1 \mathrm{ppm} /{ }^{\circ} \mathrm{C}$ over a temperature range of $-20^{\circ} \mathrm{C}$ to $100^{\circ} \mathrm{C}$. However, power dissipation was $110 \mu \mathrm{W}$. Nowadays, many researchers use derived versions of the abovementioned curvature correction technique with minor modifications.

\section{Conclusion}

Bandgap/non-bandgap based voltage reference is an important building block in analog circuit design. Evolution of voltage reference circuits is explicitly provided in chronological order to build-up a better understanding of reference circuit. We have drafted an indepth survey of classic and recent reference topologies. The design trend and current design methodologies of bandgap voltage reference have been discussed. This survey will give insight to designers while building voltage references.

\section{References}

1. Giustolisi, G. and Palumbo, G. "A detailed analysis of power-supply noise attenuation in bandgap voltage references", IEEE Transactions on Circuits and Systems I: Fundamental Theory and Applications, 50(2), pp. 185-197 (2003).

2. Widlar, R.J. "New developments in IC voltage regulators", IEEE Journal of Solid-State Circuits, 6(1), pp. 2-7 (1971).

3. Kuijk, K.E. "A precision reference voltage source", IEEE Journal of Solid-State Circuits, 8(3), pp. 222226 (1973).

4. Brokaw, A.P. "A simple three-terminal IC bandgap reference", IEEE Journal of Solid-State Circuits, 9(6), pp. 388-393 (1974).

5. Neuteboom, H., Kup, B.M. and Janssens, M. "A DSPbased hearing instrument IC", IEEE Journal of SolidState Circuits, 32(11), pp. 1790-1806 (1997).

6. Banba, H., Shiga, H., Umezawa, A., Miyaba, T., Tanzawa, T., Atsumi, S. and Sakui, K. "A CMOS bandgap reference circuit with sub-1-V operation", IEEE Journal of Solid-State Circuits, 34(5), pp. 670674 (1999).

7. Leung, K.N. and Mok, P.K. "A sub-1-V $15-\mathrm{ppm} /{ }^{\circ} \mathrm{C}$ CMOS bandgap voltage reference without requiring low threshold voltage device", IEEE Journal of SolidState Circuits, 37(4), pp. 526-530 (2002).

8. Ming-Dou, K., Jung-Sheng, C. and Ching-Yun, C. "A CMOS bandgap reference circuit for sub-1-V operation without using extra low-threshold-voltage device", IEICE Transactions on Electronics, 88(11), pp. 21502155 (2005).

9. Annema, A.J. and Goksun, G. "A $0.0025 \mathrm{~mm}^{2}$ bandgap voltage reference for $1.1 \mathrm{~V}$ supply in standard $0.16 \mu \mathrm{m}$ CMOS", In IEEE International Solid-State Circuits Conference Digest of Technical Papers, pp. 364-366 (2012).

10. Filanovsky, I. and Allam, A. "Mutual compensation of mobility and threshold voltage temperature effects with applications in CMOS circuits", IEEE Transactions on Circuits and Systems I: Fundamental Theory and Applications, 48(7), pp. 876-884 (2001).

11. Najafizadeh, L. and Filanovsky, I.M. "Towards a sub1 V CMOS voltage reference", In Proceedings of IEEE International Symposium on Circuits and Systems, 1, pp. I-53-56 (2004).

12. Leung, K.N. and Mok, P. "A CMOS voltage reference based on weighted Delta VGS for CMOS low-dropout linear regulators", Solid-State Circuits, IEEE Journal of, 38(1), pp. 146-150 (2003).

13. De Vita, G. and Iannaccone, G. "An ultra-low-power, temperature compensated voltage reference generator", In Proceedings of IEEE Custom Integrated Circuits Conference, pp. 751-754 (2005). 
14. De Vita, G., Iannaccone, G. and Andreani, P. "A 300 $\mathrm{nW}, 12 \mathrm{ppm} /{ }^{\circ} \mathrm{C}$ voltage reference in a digital 0.35 $\mu \mathrm{m}$ CMOS process", In IEEE Symposium on VLSI Circuits, pp. 81-82 (2006).

15. De Vita, G. and Iannaccone, G. "A sub-1-V, 10 $\mathrm{ppm} /{ }^{\circ} \mathrm{C}$, Nanopower voltage reference generator", IEEE Journal of Solid-State Circuits, 42(7), pp. 15361542 (2007).

16. Magnelli, L., Crupi, F., Corsonello, P., Pace, C. and Iannaccone, G. "A $2.6 \mathrm{nW}, 0.45 \mathrm{~V}$ temperaturecompensated subthreshold CMOS voltage reference", IEEE Journal of Solid-State Circuits, 46(2), pp. 465474 (2011).

17. Albano, D., Crupi, F., Cucchi, F. and Iannaccone, G. "A picopower temperature-compensated, subthreshold CMOS voltage reference", International Journal of Circuit Theory and Applications, 42(12), pp. 13061318 (2014).

18. Ytterdal, T. "CMOS bandgap voltage reference circuit for supply voltages down to $0.6 \mathrm{~V}$ ", IET Electronics Letters, 39(20), pp. 1427-1428 (2003).

19. Giustolisi, G., Palumbo, G., Criscione, M. and Cutri, F. "A low-voltage low-power voltage reference based on subthreshold MOSFETs", IEEE Journal of SolidState Circuits, 38(1), pp. 151-154 (2003).

20. Huang, P.-H., Lin, H. and Lin, Y.-T. "A simple subthreshold CMOS voltage reference circuit with channel-length modulation compensation", IEEE Transactions on Circuits and Systems II: Express Briefs, 53(9), pp. 882-885 (2006).

21. Song, B.-S. and Gray, P.R. "A precision curvaturecompensated CMOS bandgap reference", IEEE Journal of Solid-State Circuits, 18(6), pp. 634-643 (1983).

22. Lee, I., Kim, G. and Kim, W. "Exponential curvaturecompensated BiCMOS bandgap references", IEEE Journal of Solid-State Circuits, 29(11), pp. 1396-1403 (1994).

23. Rincon-Mora, G. and Allen, P.E. "A 1.1-V currentmode and piecewise-linear curvature-corrected bandgap reference", IEEE Journal of Solid-State Circuits, 33(10), pp. 1551-1554 (1998).

24. Leung, K.N., Mok, P.K. and Leung, C.Y. "A 2$\mathrm{V} 23-\mu \mathrm{A} 5.3 \mathrm{ppm} /{ }^{\circ} \mathrm{C}$ curvature-compensated CMOS bandgap voltage reference", IEEE Journal of SolidState Circuits, 38(3), pp. 561-564 (2003).

25. Gunawan, M., Meijer, G.C., Fonderie, J. and Huijsing, J.H. "A curvature-corrected low-voltage bandgap reference", IEEE Journal of Solid-State Circuits, 28(6), pp. 667-670 (1993).
26. Malcovati, P., Maloberti, F., Fiocchi, C. and Pruzzi, M. "Curvature-compensated BiCMOS bandgap with 1-V supply voltage", IEEE Journal of Solid-State Circuits, 36(7), pp. 1076-1081 (2001).

27. Mitrea, O., Popa, C., Manolescu, A. and Glesner, M. "A curvature-corrected CMOS bandgap reference", Advances in Radio Science, 1(D.2), pp. 181-184 (2005).

28. Ker, M.-D. and Chen, J.-S. "New curvature-compensation technique for CMOS bandgap reference with sub1-V operation", IEEE Transactions on Circuits and Systems II: Express Briefs, 53(8), pp. 667-671 (2006).

29. Guan, X., Wang, X., Wang, A. and Zhao, B. "A 3 V $110 \mu \mathrm{w} 3.1 \mathrm{ppm} /{ }^{\circ} \mathrm{C}$ curvature-compensated CMOS bandgap reference", Analog Integrated Circuits and Signal Processing, 62(2), pp. 113-119 (2010).

\section{Biographies}

Vinayak Hande received $\mathrm{PhD}$ degree in Electrical Engineering from Indian Institute of Technology Bombay (IIT-Bombay), India, in 2015. Before joining the $\mathrm{PhD}$ program, he worked in GDA Technologies Limited, India, as an analog IC designer. After completing $\mathrm{PhD}$, he joined the Department of Electrical Engineering as an Assistant Professor at Indian Institute of Technology Ropar (IIT-Ropar). His research areas include analog and mixed-signal circuit design for noise-tolerant system design.

Maryam Shojaei Baghini received MS and PhD degrees in Electrical Engineering from Sharif University of Technology, Tehran, Iran, in 1991 and 1999, respectively. In between, she worked in the industry as senior analog IC design engineer. In 2001, she joined Indian Institute of Technology Bombay (IIT-Bombay) as a post-doctoral fellow, where she is currently a professor in the Department of Electrical Engineering. Dr. Maryam Shojaei Baghini is author/co-author of more than 160 international journal and conference papers, inventor/co-inventor of 7 granted US patents and one issued Indian patent, and inventor/co-inventor of 34 more filed patent applications. She is also jointrecipient of 10 awards. She has served in technical committees of many conferences, including IEEE ASSCC, for several years. Her research areas include analog, mixed-signal, and RF circuit and system design for emerging applications; instrumentation and lownoise circuit and system design for sensor applications; device-circuit co-design; and energy harvesting. 